\title{
EL REGISTRO FAUNÍSTICO DEL MERCADO DE LA PUERTA DE LA CARNE DE SEVILLA (SIGLOS XV-XVII)
}

\section{THE FAUNAL ASSEMBLAGE IN THE PUERTA DE LA CARNE MARKET OF SEVILLE (15TH-17TH CENTURIES)}

\author{
ESTEBAN GARCÍA-VIÑAS \\ Laboratorio de Paleobiología. Instituto Andaluz del Patrimonio Histórico. Avda. de los Descubrimientos s/n. 41092. Sevilla \\ Correo-e: garciav.esteban@gmail.com (D) https://orcid.org/0000-0002-8607-7505 \\ ELOÍSA BERNÁLDEZ-SÁNCHEZ \\ Laboratorio de Paleobiología. Instituto Andaluz del Patrimonio Histórico. Avda. de los Descubrimientos s/n. 41092. Sevilla \\ Departamento de Sistemas Físicos, Químicos y Naturales. Universidad Pablo de Olavide. Carretera de Utrera km 1. 41013. Sevilla \\ Correo-e: eloisa.bernaldez@juntadeandalucia.es (D) https://orcid.org/0000-0002-7467-2139 \\ ÁLVARO JIMÉNEZ-SANCHO \\ Arqueópolis S.L. C/ Lumbreras, 26, 3D. 41002. Sevilla. \\ Correo-e: alv.jim@gmail.com D https://orcid.org/0000-0002-8580-7481 \\ PABLO OLIVA MUÑOZ \\ Arqueópolis S.L. C/ Lumbreras, 26, 3²D. 41002. Sevilla. \\ Correo-e: polivam@gmail.com \\ DANIELA LALLONE \\ C/Mendoza de los Ríos, 9. 41002. Sevilla. \\ Correo-e: danielalallone@hotmail.com
}

Resumen: El presente trabajo recoge el análisis paleobiológico y tafonómico del conjunto faunístico rescatado en el yacimiento arqueológico del Mercado de la Puerta de la Carne (Sevilla). La sucesión estratigráfica estudiada comienza en el s. XV coincidiendo con la construcción del Matadero y finaliza en la segunda mitad del siglo XVII. Durante este periodo la ciudad de Sevilla pasa de un máximo poblacional promovido por el comercio con América a un declive de más de la mitad de los habitantes causado por la peste negra. El registro arqueozoológico recoge rasgos de ambas situaciones, un basurero vinculado probablemente al matadero de reses que continúa en activo a lo largo de tres siglos y una acumulación de ratas negras que bien podría estar relacionada con uno de los brotes de peste que se sucedieron a lo largo de esos dos siglos de historia. Además, en esta intervención arqueológica se han realizado diferentes metodologías de rescate de material faunístico para demostrar la importancia de un rescate adecuado, como otros investigadores vienen apuntando,
Abstract: This paper talks about the palaeobiological and taphonomical analysis of a faunal assemblage registered in the Mercado de la Puerta de la Carne archaeological site (Seville). The stratigraphy studied started in the $15^{\text {th }}$ century, when the slaughterhouse was built, and finished in the second half of the $17^{\text {th }}$ century.During this period, the population of Seville was increased more than the double due to the trade connexion with America and was decreased more than a half of the population due to the black pest. In the archaeozoological register, we could find evidences of both events: a rubbish dump related to the cattle slaughterhouse and an accumulation of black rats, probably related to any of the pest outbreaks that happened in these centuries of the history. In addition, in this archaeological site we carried out three different excavation methodologies to prove the necessity of an adequate methodology to pick up the majority of the taphons, as other specialists also note. The probability to find a whole taphocoenosis increases when the archaeozoologist takes 
y cómo la participación de un especialista en la excavación aumenta la probabilidad de hallar una tafocenosis más completa, reduciendo el sesgo del registro hacia especies de mayor tamaño por la mayor visibilidad de sus restos.

Palabras clave: Matadero, Reyes Católicos, Edad Moderna, Arqueozoología, Arqueomalacología.

\section{INTRODUCCIÓN}

La historia de una ciudad se suele contar a partir de la información recogida en los archivos y de la registrada en los yacimientos arqueológicos. La basura orgánica producida por los habitantes forma parte de este registro arqueológico y contiene información acerca de la vida cotidiana y de la relación de los humanos con el medio.

Durante siglos de vida, en la ciudad de Sevilla se han producido desechos que eran arrojados extramuros o en cualquier otro lugar recogido en las ordenanzas municipales (Collantes de Terán 1984). Estos muladares o grandes basureros extramuros solían jugar un papel importante en el urbanismo de la ciudad desde varios puntos de vista. El primero de ellos está relacionado con la transformación del paisaje (Bernáldez 1996), ya que la basura puede modificar biotopos, colmatando arroyos y desniveles hasta llegar a un paisaje alomado (Cortés 1998). Uno de los grandes muladares de la ciudad de Sevilla, localizado en la actual calle Torneo, fue cedido a Hernando Colón (Arenas et al. 2004), de este modo consiguió una atalaya de basura desde la que controlar el trasiego de barcos en el río. Los muladares de extramuros de Sevilla casi siempre están relacionados con las puertas de acceso a la ciudad, ya que, a pesar de que en las ordenanzas municipales se recogía que los vertederos debían estar situados en lugares alejados de ellas (Collantes de Terán 1984), los vecinos arrojaban los carros de basura a poca distancia de esas entradas. Esta basura, cerca de las puertas, produjeron montículos que llegaron a los niveles más altos de la muralla, como cuenta Collantes de Terán (1984) que sucedía en la Puerta Real o en la Puerta de la Carne (Cortés 1998: 90). Debido a estas acumulaciones, aunque las puertas de acceso a la ciudad se cerrasen a ciertas horas de la noche, la gente no encontraba impedimento alguno para acceder a la misma puesto que solo tenía que remontar esos montículos adosados a la muralla a ambos lados de las puertas.

El segundo aspecto al que contribuyen los muladares es a la ganancia de terreno agrícola debido a la part in the excavation, reducing the registration bias towards larger species due to the greater visibility of their remains.

Keywords: Slaughterhouse, Catholic Monarchs, Modern Age, Archaeozoology, Archaeomalacology.

formación de suelos enriquecidos en nutrientes. Este podría ser el caso de la Huerta de Espantaperros, un espacio extramuros colindante con el Matadero de los Reyes Católicos, hoy denominado Mercado de la Puerta de la Carne, que se utilizaba como muladar, al menos, desde época medieval islámica (Bosch 1984; Collantes de Terán 1984). Probablemente esta huerta fue también el basurero de los despojos óseos procedentes de dicho matadero desde su construcción en el siglo XV.

El tercer aspecto que se puede considerar es el uso de los muladares y basureros como canteras de material de construcción. En algunas de las excavaciones realizadas en la ciudad se ha comprobado que los suelos se hacían con tierras que contenían una gran cantidad de restos orgánicos procedentes de la actividad trófica de los habitantes (Bernáldez y Bernáldez 2002), es decir, niveles de basura que por su horizontalidad demostraban que no eran basureros de primera formación sino de reciclaje. En algunos de ellos se encontraron grandes valvas de ostras planas (Ostrea edulis), las mismas que pueden observarse intencionalmente colocadas entre los sillares de la Catedral o en el edificio de la Universidad de Sevilla de la calle San Fernando. De este modo la basura parte de ser un problema de espacio y salubridad a convertirse en un material de construcción.

Atendiendo a estos aspectos se ha realizado la intervención paleobiológica en la excavación arqueológica del Mercado de la Puerta de la Carne. Una excavación que ha dejado al descubierto parte del antiguo matadero construido durante el reinado de los Reyes Católicos en 1489 (Collantes de Terán 1984). Este edificio funcionó como matadero de ganado hasta 1914, año en el que fue reemplazado por un mercado de abastos (Jiménez 2017), lo que supone 500 años de historia cotidiana en unos muros y cimientos conservados junto a la basura sobre la que se construyó. Una basura que no sólo sirvió de base para la construcción del matadero, sino que se utilizó en las reparaciones y remodelaciones que se hicieron a lo largo de su existencia para aumentar la cota de la calle colindante. Esto se deduce de la estratigrafía de las catas excavadas para el estudio 
paleobiológico donde la horizontalidad de los estratos y su contenido muestran que la nivelación y el recrecimiento del terreno se hizo con basura, posiblemente de los muladares próximos al matadero. El análisis del contenido orgánico (conchas y huesos) ha proporcionado una interesante información sobre el uso de los muladares como cantera de construcción, pero también de las costumbres cotidianas de nuestros antepasados y de las características biológicas de algunos de los recursos naturales que explotaban.

\section{DESCRIPCIÓN ESTRATIGRÁFICA}

El solar donde se sitúa el Mercado de la Puerta de la Carne de Sevilla presenta un elevado interés histórico en el que la Paleobiología tiene un papel importante debido a su localización en antiguos muladares (con huesos y conchas) a extramuros de la ciudad (fig. 1).

En dicho solar se seleccionó un área al sur del antiguo matadero donde la secuencia estratigráfica registrada era casi homogénea y en ella se realizó una cata, la cual se excavó en dos cortes (contiguos) con el objetivo de llevar a cabo un contraste de metodologías de rescate de material faunístico. El Corte A, que presentaba una superficie de $186 \mathrm{~cm}$ por $66 \mathrm{~cm}$, fue excavado de manera sistemática para rescatar todo el material faunístico procedente de la misma. En este corte se han documentado las unidades estratigráficas 22, 26, 29, 48, 30 y 55 . La unidad estratigráfica 55 no se excavó en toda la longitud del corte porque se detectó una zona que podría haber sido removida con posterioridad, por lo tanto las dimensiones se redujeron a una superficie de $101 \mathrm{~cm}$ por $66 \mathrm{~cm}$. El Corte B se planteó con una superficie de 100 $\mathrm{cm}$ por $33 \mathrm{~cm}$. En este se pudo cribar la tierra de todos los estratos anteriormente citados con la excepción de la UE 29, cuyo registro no se conservaba en este corte. A continuación se presenta una descripción somera de cada uno de esos estratos intervenidos (fig. 2).

- UE 22: Enlosado tipo Sardinel (potencia: $12 \mathrm{~cm}$, incluye la anchura de los ladrillos y la argamasa). Datado en el siglo XVII.

- UE 26: Primer nivel horizontal (potencia: $67 \mathrm{~cm}$ ). Color oscuro y textura arcillosa. Presenta fauna, cerámica y metal. Datado en la segunda mitad del s. XVII.

- UE 27: Segundo nivel horizontal (potencia: $28 \mathrm{~cm}$ ). Color blanquecino y alto contenido en arena. Es un nivel de escombros. Presenta menos material faunístico que el anterior. Datado en la primera mitad del s. XVII.
- UE 29: Tercer nivel horizontal (potencia: de $22 \mathrm{~cm}$ a $15 \mathrm{~cm}$ ). Color rojizo y alto contenido en arena. Material de construcción con alguna teja y cantos rodados. Presenta escaso material faunístico en referencia al anterior. Datado en la primera mitad del s. XVII.

- UE 48: Nivel con buzamiento (potencia: $37 \mathrm{~cm}$ ). Depósito pegado al muro con color oscuro y arcilloso. Presenta restos de fauna sobre todo en la parte más baja que está en contacto con el suelo de cal (UE 30). Inicios del s. XVII.

- UE 30: Capa de cal (potencia: $7 \mathrm{~cm}$ ). Finales del s. XV.

- UE 55: Unidad de relleno de color oscuro y textura arcillosa. Relleno de la fosa 76. Sólo se ha excavado una potencia de $30 \mathrm{~cm}$, aunque la potencia del estrato es de $90 \mathrm{~cm}$. Final del s. XV.

\section{METODOLOGÍA}

\subsection{Información biológica}

El análisis paleobiológico comenzó por la determinación de la parte anatómica y de la especie a la que pertenece un elemento. Para ello, se utilizaron tanto la bibliografía especializada como las colecciones de referencia osteológica y malacológica del Instituto Andaluz del Patrimonio Histórico (IAPH) y de la Estación Biológica de Doñana (EBD). Todo el material que no pudo ser determinado a nivel específico, fue clasificado a nivel de género o familia o dentro del grupo de los macroungulados (Tipo I con más de 200 $\mathrm{kg}$ ), mesoungulados (especies de mamíferos Tipo II, de 18 a $200 \mathrm{~kg}$ ) y microvertebrados (Tipo III, menos de $18 \mathrm{~kg}$ y Tipo IV, menos de $1 \mathrm{~kg}$ ). Esta catalogación de las especies atendiendo a la masa corporal fue propuesta por Bernáldez $(1996,2011)$ a partir de los resultados de un estudio bioestratinómico desarrollado en el Parque Nacional de Doñana, ya que las carcasas muestran diferentes pautas de conservación esquelética en función de la masa corporal de los ejemplares. Por último, se clasificaron como indeterminadas aquellas esquirlas óseas sin posibilidad de adscripción anatómica ni taxonómica. La estimación de la edad de los individuos sacrificados se realizó a partir de la fusión de las epífisis de los huesos y de la erupción de las piezas dentales (Barone 1999; Schmid 1972), posteriormente se clasificaron los individuos siguiendo a Morales et al. (1994: 38) y Riquelme (1998). 


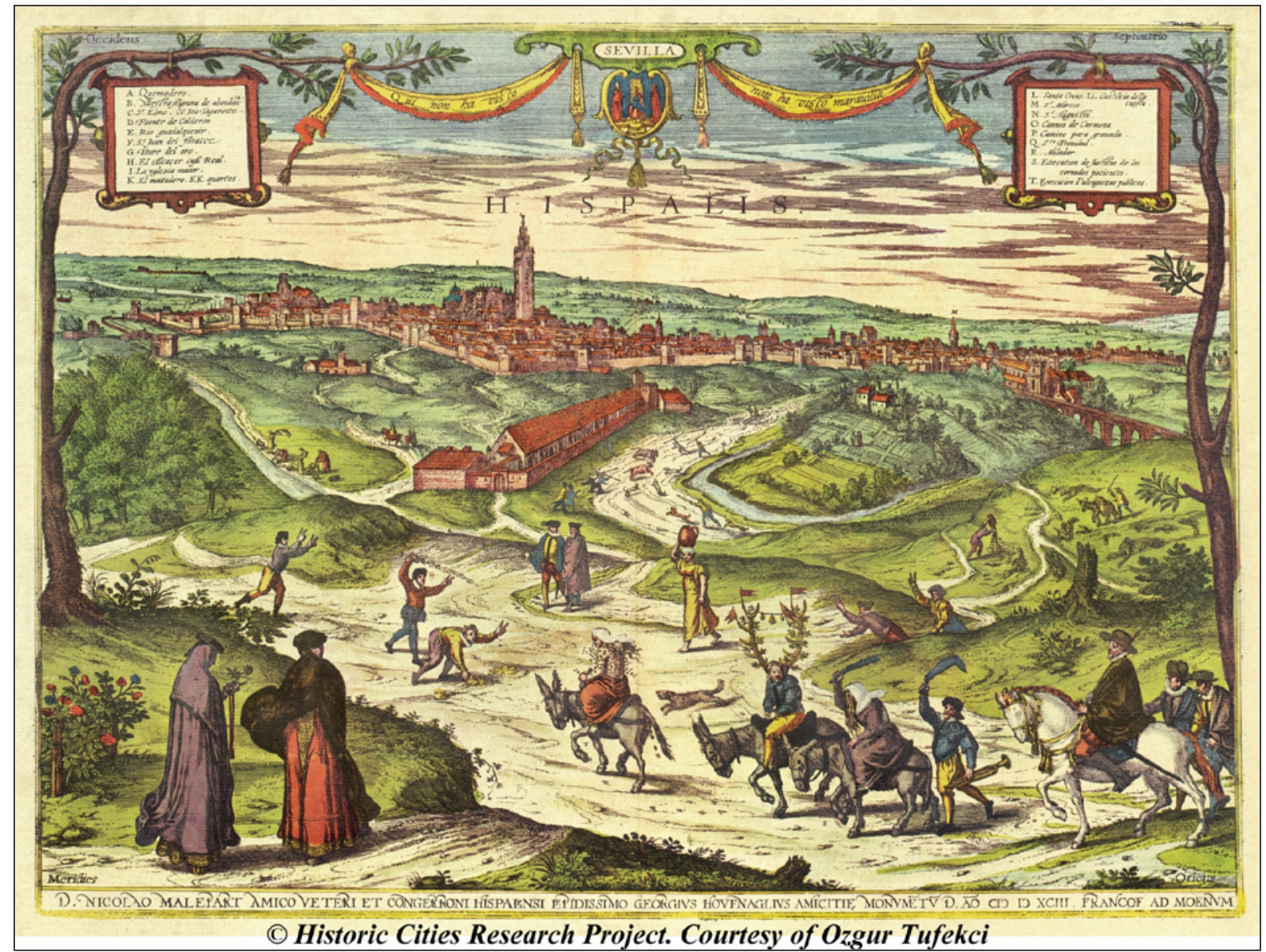

Figura 1. Situación del Mercado de la Puerta de la Carne extramuros de la ciudad de Sevilla (edificio alargado localizado en el centro de la imagen). Imagen procedente de Civitates Orbis Terrarum (1593)1.

\subsection{Información tafonómica}

En este análisis, además de estudiar las huellas de uso y erosión (Lyman 1994; Fisher 1995; Yravedra 2006), se trató la tafonomía del depósito y del conjunto orgánico atendiendo a los criterios biostratinómicos propuestos por Bernáldez (2009; 2011). Se analizó el estado de conservación del registro óseo a partir del índice de fragmentación (IF) descrito por Bernáldez y Bernáldez (2000) y la conservación del conjunto esquelético a partir del índice de conservación esquelética (SCI) definido por Bernáldez (1996). Por un lado, SCI se calcula comparando el número de huesos que se encuentran en un yacimiento por especie (NISP) con el que presenta un individuo vivo de esa misma especie (NS; Bernáldez 2009) y por otro lado, IF se calcula a partir del número de restos totales

1. Historic Cities Research Project. Courtesy of Ozgur Tufekci.
(NRT) y el número de huesos determinados (NISP). Ambos índices se han calculado únicamente a partir de los datos aportados por ejemplares Tipos I y II, excluyendo el número de piezas dentales aisladas.

$-\mathrm{SCI}=(\mathrm{NISP} /(\mathrm{NMI} * \mathrm{NS})) * 100$
$-\mathrm{IF}=\log (\mathrm{NRT} / \mathrm{NISP})$

Por último, la tafonomía del depósito se estudió teniendo en cuenta el volumen de cada estrato, ya que la abundancia de individuos (NMI) y especímenes identificados (NISP), así como la riqueza faunística, puede estar relacionada con las dimensiones del mismo (Bernáldez y Bernáldez 2000; García-Viñas y Bernáldez 2013). Por este motivo la acumulación de material orgánico fue estudiada a partir de la densidad de restos totales (DRT), de especímenes (DISP), de masa (DM) y de individuos (DI) que contenían las distintas unidades estratigráficas. 


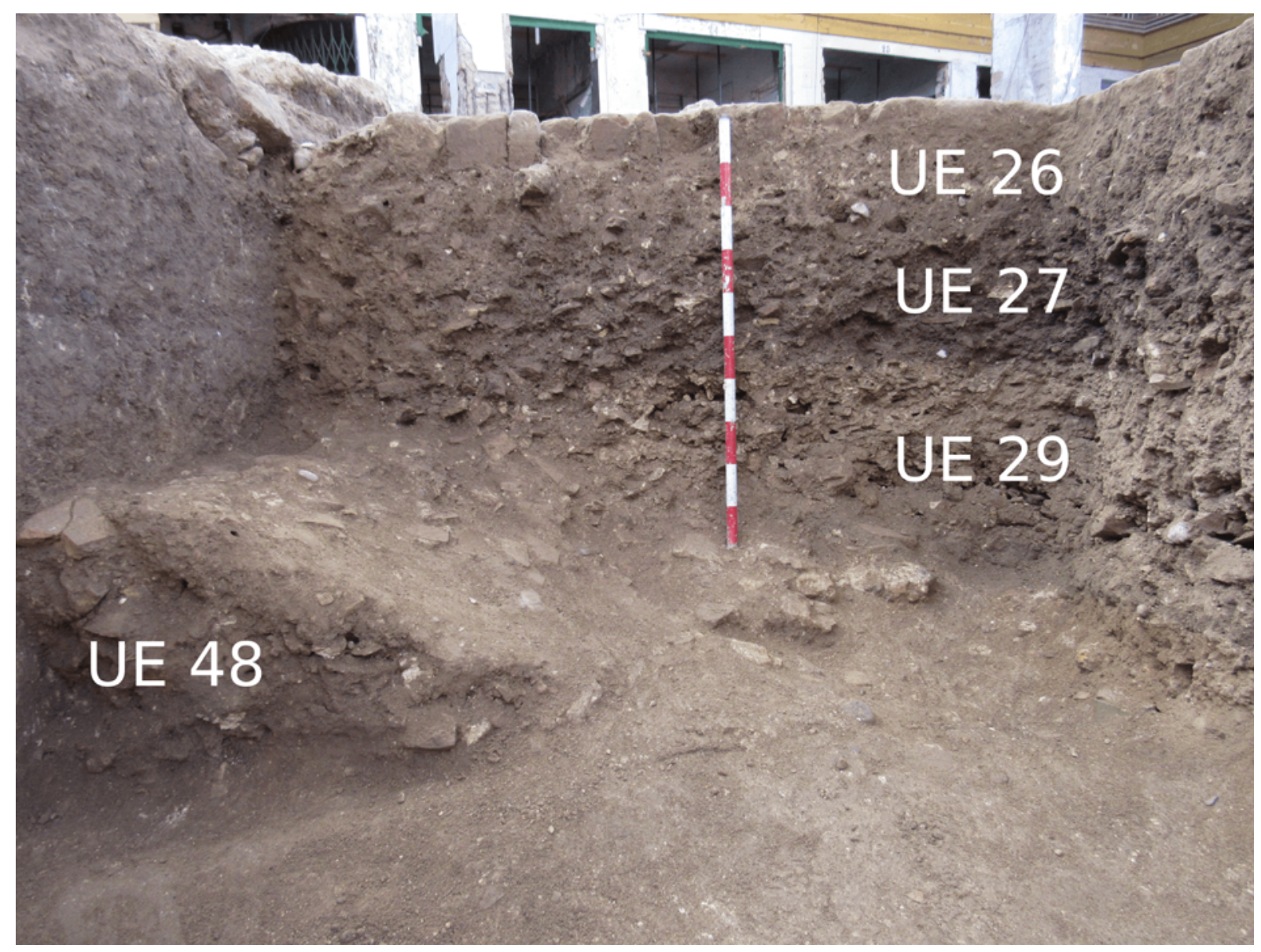

Figura 2. Detalle de la secuencia estratigráfica excavada en el Corte A. Se puede apreciar la tendencia a la horizontalidad de todos los estratos, excepto la UE 48.

\section{RESULTADOS}

\subsection{Características biológicas del registro}

El total de material faunístico analizado en la campaña de excavación del año 2017 suma 2.960 restos (2.724 excluyendo las piezas dentales), $13,76 \mathrm{~kg}$ y 684 ejemplares, de los que los vertebrados suponen el $77 \%$ de los elementos, el $88 \%$ de la masa y $19 \%$ de los individuos.

La cata intervenida contiene 2.778 elementos con $8,20 \mathrm{~kg}$ de, al menos, 643 individuos. En este caso, los vertebrados están representados por un $75 \%$ de los restos, un $93 \%$ de la masa y un $14 \%$ de los ejemplares. En concreto se han determinado 92 ejemplares de vertebrados (tabla 1): 14 Bos taurus (vaca), un Equus caballus (caballo), siete Sus sp. (probablemente cerdo), 12 caprinos (cabra/oveja), un Canis familiaris (perro), dos Felis catus (gato), tres Oryctolagus cuniculus (conejo), un Rattus norvegicus (rata gris), 26 Rattus rattus (rata negra), un roedor indeterminado, cinco Gallus domesticus (gallo), cinco aves indeterminadas, ocho peces indeterminados y dos anuros (ranas y sapos). Además se ha descrito un molar de Homo sapiens. Los suidos determinados podrían tratarse de cerdos atendiendo a su biometría. Los valores de GLl (Longitud máxima de la vista lateral) de dos astrágalos de suido estudiados son inferiores a $40 \mathrm{~mm}$, por lo que según los resultados de Rowley-Conwy et al. (2012) es probable que pertenecieran a cerdos. De todos modos hay que tener en cuenta que estos resultados no son del todo válidos para ejemplares del sur de la península ibérica. Por su parte, el registro malacológico está compuesto por 551 individuos: 21 Ostrea edulis (ostra plana), un Pecten maximus (vieira), seis Ruditapes decussatus (almeja fina), un Cerastoderma edulis (berberecho), dos ejemplares de la familia Unionidae, 
Tabla 1. Registro faunístico de vertebrados rescatado en los Cortes A y B. Siendo NR el número de restos contabilizando las piezas dentales, NISP el número de especímenes identificados por taxón sin contabilizar las piezas dentales, M la masa y NMI el número mínimo de individuos. La abreviación Macro. corresponde a macroungulados, Meso. a mesoungulados, Micro. a microvertebrados e Indet. a indeterminados.

\begin{tabular}{|c|c|c|c|c|c|c|c|c|}
\hline Especie & & UE 22 & UE 26 & UE 27 & UE 29 & UE 48 & UE 55 & Total \\
\hline \multirow{4}{*}{ Bos taurus } & NR & 6 & 51 & 27 & 5 & 53 & 136 & 276 \\
\hline & NISP & 3 & 42 & 9 & 1 & 30 & 61 & 146 \\
\hline & M & 131 & 1364 & 560 & 25 & 1765 & 962 & 4807 \\
\hline & NMI & 1 & 3 & 2 & 1 & 5 & 2 & 14 \\
\hline \multirow{3}{*}{ Equus caballus } & NISP & - & 1 & - & - & - & - & 1 \\
\hline & M & - & 54 & - & - & - & - & 54 \\
\hline & NMI & - & 1 & - & - & - & - & 1 \\
\hline \multirow{4}{*}{ Suido } & NR & 1 & 8 & 2 & 1 & 2 & 4 & 18 \\
\hline & NISP & 1 & 4 & 2 & 1 & 1 & 4 & 13 \\
\hline & M & 5 & 39 & 30 & 4 & 2 & 8 & 88 \\
\hline & NMI & 1 & 1 & 1 & 1 & 1 & 2 & 7 \\
\hline \multirow{4}{*}{ Caprino } & NR & 1 & 74 & 12 & 5 & 16 & 27 & 135 \\
\hline & NISP & 1 & 54 & 10 & 4 & 8 & 17 & 94 \\
\hline & M & 6 & 380 & 65,52 & 15 & 43 & 101 & 610,52 \\
\hline & NMI & 1 & 5 & 2 & 1 & 1 & 2 & 12 \\
\hline \multirow{3}{*}{ Canis familiaris } & NISP & - & - & - & - & - & 1 & 1 \\
\hline & M & - & - & - & - & - & 5 & 5 \\
\hline & NMI & - & - & - & - & - & 1 & 1 \\
\hline \multirow{3}{*}{ Felis catus } & NISP & - & 2 & - & - & - & 1 & 3 \\
\hline & M & - & 2,72 & - & - & - & 2 & 4,72 \\
\hline & NMI & - & 1 & - & - & - & 1 & 2 \\
\hline \multirow{3}{*}{ Oryctolagus cuniculus } & NISP & - & 7 & 1 & - & 2 & - & 10 \\
\hline & M & - & 6,25 & 0,99 & - & 0,51 & - & 7,75 \\
\hline & NMI & - & 1 & 1 & - & 1 & - & 3 \\
\hline \multirow{3}{*}{ Rattus norvegicus } & NISP & - & 4 & - & - & - & - & 4 \\
\hline & M & - & 1,9 & - & - & - & - & 1,9 \\
\hline & NMI & - & 1 & - & - & - & - & 1 \\
\hline \multirow{4}{*}{ Rattus rattus } & NR & - & 47 & 41 & - & 112 & 1 & 201 \\
\hline & NISP & - & 42 & 36 & - & 105 & 1 & 184 \\
\hline & M & - & 5,14 & 4,39 & - & 14,35 & 0,17 & 24,05 \\
\hline & NMI & - & 6 & 6 & - & 13 & 1 & 26 \\
\hline
\end{tabular}




\begin{tabular}{|c|c|c|c|c|c|c|c|c|}
\hline Especie & & UE 22 & UE 26 & UE 27 & UE 29 & UE 48 & UE 55 & Total \\
\hline \multirow{3}{*}{ Roedor } & NISP & - & - & - & - & 1 & - & 1 \\
\hline & M & - & - & - & - & 0,01 & - & 0,01 \\
\hline & NMI & 一 & - & - & - & 1 & - & 1 \\
\hline \multirow{3}{*}{ Gallus domesticus } & NISP & - & 11 & 3 & - & 14 & - & 28 \\
\hline & M & - & 20,02 & 31,1 & - & 3,18 & - & 54,3 \\
\hline & NMI & - & 2 & 1 & - & 1 & - & 4 \\
\hline \multirow{3}{*}{ Ave } & NISP & - & 5 & 1 & 3 & 1 & - & 10 \\
\hline & M & - & 5,08 & 0,14 & 4,93 & 0,38 & - & 10,53 \\
\hline & NMI & - & 2 & 1 & 1 & 1 & - & 5 \\
\hline \multirow{3}{*}{ Anuro } & NISP & 1 & 一 & - & - & - & 1 & 2 \\
\hline & M & 0,2 & - & - & - & - & 0,02 & 0,22 \\
\hline & NMI & 1 & - & - & - & - & 1 & 2 \\
\hline \multirow{3}{*}{$\mathrm{Pez}$} & NISP & - & 31 & 15 & 1 & 19 & - & 66 \\
\hline & M & - & 8,79 & 7,03 & 0,69 & 1,72 & - & 18,23 \\
\hline & NMI & - & 3 & 2 & 1 & 2 & - & 8 \\
\hline \multirow{2}{*}{ Macromamífero } & NISP & - & 35 & 2 & - & 6 & - & 43 \\
\hline & M & - & 149 & 29 & - & 69 & - & 247 \\
\hline \multirow{2}{*}{ Mesomamífero } & NISP & 2 & 216 & 49 & - & 21 & 74 & 362 \\
\hline & M & 2 & 389 & 82 & - & 37 & 123 & 633 \\
\hline \multirow{2}{*}{ Micromamífero } & NISP & - & 97 & 24 & - & 50 & 9 & 180 \\
\hline & M & - & 10,88 & 6,61 & - & 5,86 & 4,84 & 28,19 \\
\hline \multirow{2}{*}{ Indeterminado } & NR & 3 & 264 & 43 & 5 & 132 & 400 & 847 \\
\hline & M & 2 & 449,31 & 142 & 25 & 161 & 368,54 & 1147,85 \\
\hline
\end{tabular}

13 Melanopsis cariosa, dos Physa acuta, nueve Theba pisana, 26 Xerotricha apicina, cinco Cochlicella acuta, ocho Rumina decollata, 27 Ferussacia folliculus, 343 Cecilioides acicula, 14 Caracollina lenticula, 67 Mediterranea hydatina y cuatro moluscos indeterminados (tabla 2).

En lo que respecta a la representación de ungulados por estrato, cabe destacar la similitud en NMI de bovinos y caprinos, con dos excepciones: en la UE 26 donde el número de caprinos duplica al de bovinos y en la UE 48 donde ocurre justo lo contrario (fig. 3). Por su parte, cuando se han determinado restos de suidos y équidos, estos quedan representados por un único ejemplar. Por último, en lo que respecta a las características biológicas de los ungulados cabe destacar que la edad de la mayor parte de los bovinos determinados se encuentra entre los 24-60 meses (subadultos; tabla 3) y de los suidos y caprinos por debajo de los 24 meses (juveniles).

Las unidades estratigráficas que no estaban incluidas en la cata suman 182 restos con 5,56 kg de, al menos, 41 ejemplares de los que los vertebrados suponen un $78 \%$ de los restos, el $79 \%$ de la masa y el $55 \%$ de los individuos. Se han descrito 22 individuos de vertebrados (tabla 4): 11 Bos taurus, un Equus caballus, dos suidos, seis caprinos, un Oryctolagus cuniculus y un ave indeterminada. El registro malacológico está compuesto por 19 ejemplares de Ostrea edulis (tabla 5). 
Tabla 2. Registro faunístico de moluscos rescatado en la cata. Siendo NR el número de restos, NISP el número de especímenes identificados por taxón, M la masa y NMI el número mínimo de individuos. La abreviación Indet. corresponde a indeterminados.

\begin{tabular}{|c|c|c|c|c|c|c|c|}
\hline Especie & & UE 26 & UE 27 & UE 29 & UE 48 & UE 55 & Total \\
\hline \multirow{3}{*}{ Ostrea edulis } & NISP & 17 & 5 & 1 & 3 & 19 & 45 \\
\hline & M & 175 & 82 & 45 & 22 & 100,4 & 424,4 \\
\hline & NMI & 11 & 3 & 1 & 2 & 4 & 21 \\
\hline \multirow{3}{*}{ Pecten maximus } & NISP & 1 & - & - & - & - & 1 \\
\hline & $\mathrm{M}$ & 5,6 & - & - & - & - & 5,6 \\
\hline & NMI & 1 & - & - & - & - & 1 \\
\hline \multirow{3}{*}{ Ruditapes decussatus } & NISP & 6 & 1 & - & - & 2 & 9 \\
\hline & M & 9,8 & 0,42 & - & - & 0,26 & 10,48 \\
\hline & NMI & 4 & 1 & - & - & 1 & 6 \\
\hline \multirow{3}{*}{ Cerastoderma edulis } & NISP & 1 & - & - & - & - & 1 \\
\hline & M & 0,63 & - & - & - & - & 0,63 \\
\hline & NMI & 1 & - & - & - & - & 1 \\
\hline \multirow{3}{*}{ Unionidae } & NISP & 6 & - & - & - & - & 6 \\
\hline & M & 5,45 & - & - & - & - & 5,45 \\
\hline & NMI & 2 & - & - & - & - & 2 \\
\hline \multirow{3}{*}{ Melanopsis cariosa } & NISP & 12 & 1 & - & - & - & 13 \\
\hline & $\mathrm{M}$ & 6,91 & 1,15 & - & - & - & 8,06 \\
\hline & NMI & 12 & 1 & - & - & - & 13 \\
\hline \multirow{3}{*}{ Physa acuta } & NISP & 2 & - & - & - & - & 2 \\
\hline & $\mathrm{M}$ & 0,1 & - & - & - & - & 0,1 \\
\hline & NMI & 2 & - & - & - & - & 2 \\
\hline \multirow{3}{*}{ Theba pisana } & NISP & 6 & 2 & - & 2 & 2 & 12 \\
\hline & $\mathrm{M}$ & 2,28 & 0,15 & - & 0,46 & 0,49 & 3,38 \\
\hline & NMI & 6 & 1 & - & 2 & 2 & 11 \\
\hline \multirow{3}{*}{ Xerotricha apicina } & NISP & 7 & - & - & 4 & 15 & 26 \\
\hline & M & 0,5 & - & - & 0,15 & 0,82 & 1,47 \\
\hline & NMI & 7 & - & - & 4 & 15 & 26 \\
\hline \multirow{3}{*}{ Cochlicella acuta } & NISP & 2 & 1 & - & - & 2 & 5 \\
\hline & $\mathrm{M}$ & 0,18 & 0,09 & - & - & 0,17 & 0,44 \\
\hline & NMI & 2 & 1 & - & - & 2 & 5 \\
\hline \multirow{3}{*}{ Rumina decollata } & NISP & 2 & 1 & - & - & 5 & 8 \\
\hline & M & 1,2 & 0,09 & - & - & 0,3 & 1,59 \\
\hline & NMI & 2 & 1 & - & - & 5 & 8 \\
\hline
\end{tabular}




\begin{tabular}{|c|c|c|c|c|c|c|c|}
\hline Especie & & UE 26 & UE 27 & UE 29 & UE 48 & UE 55 & Total \\
\hline \multirow{3}{*}{ Ferussacia folliculus } & NISP & 20 & 7 & - & 3 & - & 30 \\
\hline & $\mathrm{M}$ & 0,42 & 0,17 & - & 0,04 & - & 0,63 \\
\hline & NMI & 20 & 4 & - & 3 & - & 27 \\
\hline \multirow{3}{*}{ Cecilioides acicula } & NISP & 205 & 115 & - & 23 & - & 343 \\
\hline & $\mathrm{M}$ & 0,83 & 0,47 & - & 0,03 & - & 1,33 \\
\hline & NMI & 205 & 115 & - & 23 & - & 343 \\
\hline \multirow{3}{*}{ Caracollina lenticula } & NISP & 6 & 4 & - & 4 & - & 14 \\
\hline & $\mathrm{M}$ & 0,28 & 0,14 & - & 0,03 & - & 0,45 \\
\hline & NMI & 6 & 4 & - & 4 & - & 14 \\
\hline \multirow{3}{*}{ Mediterranea hydatina } & NISP & 40 & 27 & - & - & - & 67 \\
\hline & $\mathrm{M}$ & 0,67 & 0,36 & - & - & - & 1,03 \\
\hline & NMI & 40 & 27 & - & - & - & 67 \\
\hline \multirow{3}{*}{ Indeterminado } & NR & 5 & 1 & - & - & - & 6 \\
\hline & $\mathrm{M}$ & 0,19 & 0,041 & - & - & - & 0,231 \\
\hline & NMI & 3 & 1 & - & - & - & 4 \\
\hline
\end{tabular}

Tabla 3. Edad de sacrificio estimada para los ungulados registrados en el Mercado de la Puerta de la Carne clasificados por rango de edad según Morales et al. (1994: 38) y Riquelme (1998).

\begin{tabular}{|c|c|c|c|c|c|c|c|}
\hline UE & Especie & Infantil & Juvenil & Subadulto & Adulto & Senil & Indet. \\
\hline \multirow{3}{*}{ UE 22} & Bos taurus & - & 1 & - & - & - & - \\
\hline & Sus sp. & - & 1 & - & - & - & - \\
\hline & Caprino & - & - & - & - & - & 1 \\
\hline \multirow{4}{*}{ UE 26} & Bos taurus & - & 2 & 1 & - & - & - \\
\hline & Equus caballus & - & 1 & - & - & - & - \\
\hline & Sus sp. & - & 1 & - & - & - & - \\
\hline & Caprino & 2 & 2 & - & - & - & 1 \\
\hline \multirow{3}{*}{ UE 27} & Bos taurus & - & - & 2 & - & - & - \\
\hline & Sus sp. & - & 1 & - & - & - & - \\
\hline & Caprino & - & 2 & - & - & - & - \\
\hline \multirow{3}{*}{ UE 29} & Bos taurus & - & - & - & - & - & 1 \\
\hline & Sus sp. & - & - & - & - & - & 1 \\
\hline & Caprino & - & 1 & - & - & - & - \\
\hline \multirow{3}{*}{ UE 48} & Bos taurus & - & - & 4 & - & - & 1 \\
\hline & Sus sp. & - & - & - & - & - & 1 \\
\hline & Caprino & - & 1 & - & - & - & - \\
\hline \multirow{3}{*}{ UE 55} & Bos taurus & - & - & 1 & - & - & 1 \\
\hline & Sus sp. & 1 & 1 & - & - & - & - \\
\hline & Caprino & - & 1 & - & - & - & 1 \\
\hline
\end{tabular}


Tabla 4. Registro faunístico de vertebrados rescatado en las unidades estratigráficas no vinculadas al corte intervenido. Siendo NR el número de restos contabilizando las piezas dentales, NISP el número de especímenes identificados por taxón sin contabilizar las piezas dentales, M la masa y NMI el número mínimo de individuos. La abreviación Indet. corresponde a indeterminados.

\begin{tabular}{|c|c|c|c|c|c|c|c|}
\hline Especie & & UE 26 & UE 27 & UE 40 & UE 46 & UE 55 & Total \\
\hline \multirow{4}{*}{ Bos taurus } & NR & 39 & 3 & 8 & 2 & 52 & 104 \\
\hline & NISP & 26 & 2 & 8 & 2 & 28 & 66 \\
\hline & M & 1124 & 192 & 415 & 67 & 1883 & 3681 \\
\hline & NMI & 4 & 1 & 1 & 1 & 4 & 11 \\
\hline \multirow{3}{*}{ Equus caballus } & NISP & 1 & - & - & - & - & 1 \\
\hline & M & 254 & - & - & - & - & 254 \\
\hline & NMI & 1 & - & - & - & - & 1 \\
\hline \multirow{3}{*}{ Suido } & NISP & - & 1 & 1 & - & - & 2 \\
\hline & M & - & 16 & 21 & - & - & 37 \\
\hline & NMI & - & 1 & 1 & - & - & 2 \\
\hline \multirow{4}{*}{ Caprino } & NR & 6 & 1 & 8 & - & - & 15 \\
\hline & NISP & 6 & 1 & 5 & - & - & 12 \\
\hline & M & 68 & 7 & 193 & - & - & 268 \\
\hline & NMI & 2 & 1 & 3 & - & - & 6 \\
\hline \multirow{3}{*}{ Oryctolagus cuniculus } & NISP & - & - & 1 & - & - & 1 \\
\hline & M & - & - & 2 & - & - & 2 \\
\hline & NMI & - & - & 1 & - & - & 1 \\
\hline \multirow{3}{*}{ Ave } & NISP & 2 & - & - & - & - & 2 \\
\hline & M & 3 & - & - & - & - & 3 \\
\hline & NMI & 1 & - & - & - & - & 1 \\
\hline \multirow{2}{*}{ Macromamífero } & NISP & 6 & - & 1 & - & - & 7 \\
\hline & M & 39 & - & 15 & - & - & 54 \\
\hline \multirow{2}{*}{ Mesomamífero } & NISP & 8 & 2 & 2 & - & - & 12 \\
\hline & M & 23 & 18 & 15 & - & - & 56 \\
\hline \multirow{2}{*}{ Indeterminado } & NR & 3 & - & 4 & - & - & 7 \\
\hline & $\mathrm{M}$ & 10 & - & 10 & - & - & 20 \\
\hline
\end{tabular}


Tabla 5. Registro faunístico de vertebrados rescatado en las unidades estratigráficas no vinculadas al corte intervenido. Siendo NIPS el número de especímenes identificados por taxón, M la masa y NMI el número mínimo de individuos.

\begin{tabular}{|c|l|c|c|c|c|c|c|}
\hline \multicolumn{1}{|c|}{ Especies } & & UE 26 & UE 27 & UE 40 & UE 46 & UE 55 & Total \\
\hline \multirow{3}{*}{ Ostrea edulis } & NISP & 3 & 13 & - & 10 & 5 & 31 \\
\cline { 2 - 10 } & $\mathrm{M}$ & 91 & 523 & - & 346 & 209 & 1169 \\
\cline { 2 - 10 } & NMI & 2 & 8 & - & 5 & 4 & 19 \\
\hline
\end{tabular}

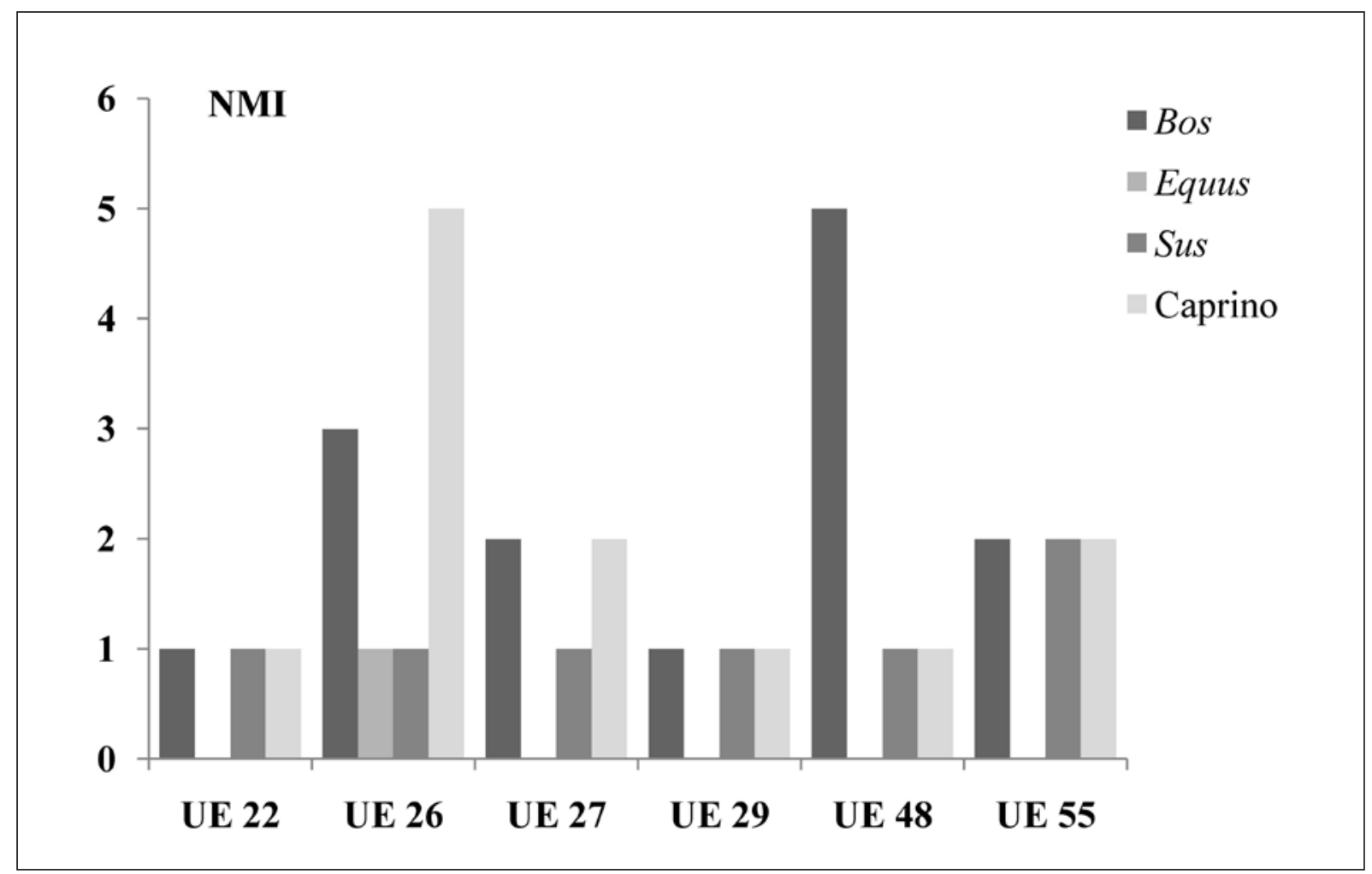

Figura 3. Número mínimo de individuos (NMI) de ungulados por unidad estratigráfica. Se observa que el NMI de bovinos y caprinos es superior al de suidos y caballos y similar en todos los estratos, excepto en la UE 26 donde dominan los caprinos y la UE 48 donde lo hacen los bovinos.

\subsection{Características tafonómicas del registro}

Los aspectos tafonómicos que serán tratados en este análisis abarcan desde el tafón (fragmento de hueso o concha) como elemento individual hasta la asociación faunística en comparación con un modelo natural descrito por Bernáldez et al. (2017).

El estado de conservación de los restos e individuos ha sido descrito a partir de los índices de conservación esquelética (SCI) y de fragmentación (IF). Por un lado, los valores de SCI están siempre por debajo del 5\% en cada una de las especies de ungulados (tabla 6), no observándose diferencias entre los estratos analizados. Dichos valores van en concordancia con lo obtenido en otros yacimientos arqueológicos y con el potencial fósil propuesto por Behrensmeyer y Boaz (1989) en un ecosistema sin intervención humana, donde se estimó que solo el 5\% de los huesos tendrían posibilidades de permanecer en el registro fósil. Es decir, los porcentajes de conservación esquelética obtenidos en el yacimiento del Mercado de la Puerta de la Carne permiten confirmar 
Tabla 6. Valores del índice de conservación esquelética (SCI) calculado para los ungulados.

\begin{tabular}{|l|c|c|c|c|}
\hline & Bos taurus & Equus caballus & Sus sp. & Caprino \\
\hline UE 22 & 1,46 & - & 0,37 & 0,49 \\
\hline UE 26 & 4,39 & 0,50 & 1,49 & 5,27 \\
\hline UE 27 & 2,20 & - & 0,74 & 2,44 \\
\hline UE 29 & 0,49 & - & 0,37 & 1,95 \\
\hline UE 48 & 2,93 & - & 0,37 & 3,90 \\
\hline UE 55 & 1,71 & - & 0,74 & 4,15 \\
\hline
\end{tabular}

Tabla 7. Número de huesos por parte anatómica de las especies de ungulados más comunes en los estratos de la cata intervenida.

\begin{tabular}{|c|c|c|c|c|c|c|c|c|c|c|c|c|c|c|c|}
\hline & \multicolumn{5}{|c|}{ Bos taurus } & \multicolumn{5}{|c|}{ Sus sp. } & \multicolumn{5}{|c|}{ Ovis/Capra } \\
\hline & $\begin{array}{l}\text { UE } \\
26\end{array}$ & $\begin{array}{l}\text { UE } \\
27\end{array}$ & $\begin{array}{l}\text { UE } \\
29\end{array}$ & $\begin{array}{c}\text { UE } \\
48\end{array}$ & $\begin{array}{c}\text { UE } \\
55\end{array}$ & $\begin{array}{l}\text { UE } \\
26\end{array}$ & $\begin{array}{l}\text { UE } \\
27\end{array}$ & $\begin{array}{l}\text { UE } \\
29\end{array}$ & $\begin{array}{c}\text { UE } \\
48\end{array}$ & $\begin{array}{l}\text { UE } \\
55\end{array}$ & $\begin{array}{l}\text { UE } \\
26\end{array}$ & $\begin{array}{l}\text { UE } \\
27\end{array}$ & $\begin{array}{l}\text { UE } \\
29\end{array}$ & $\begin{array}{l}\text { UE } \\
48\end{array}$ & $\begin{array}{l}\text { UE } \\
55\end{array}$ \\
\hline Premolar maxilar & & - & - & - & 3 & - & - & - & - & - & - & - & 一 & - & \\
\hline Molar maxilar & 1 & - & - & 2 & - & - & - & - & - & - & - & - & - & - & 1 \\
\hline Incisivo mandíbula & 1 & - & - & 1 & 3 & 1 & - & - & - & - & - & - & - & 1 & 1 \\
\hline Canino mandíbula & & - & - & - & - & - & - & - & 1 & - & - & - & - & - & - \\
\hline Premolar mandíbula & 3 & - & 4 & 2 & 7 & 1 & - & - & - & - & 4 & - & - & 1 & 一 \\
\hline Molar mandíbula & 1 & 2 & 1 & 5 & 9 & - & - & - & 1 & - & 8 & 3 & - & 3 & 3 \\
\hline Pieza dental & 3 & 17 & - & 13 & 53 & 2 & - & - & - & - & 8 & - & 1 & 5 & 5 \\
\hline Clavija & 2 & - & - & 1 & - & - & - & - & - & - & - & - & 1 & - & - \\
\hline Cráneo & 16 & - & - & 1 & 2 & 1 & - & - & - & - & 2 & - & - & - & - \\
\hline Mandíbula & 2 & 7 & - & 25 & 57 & 1 & - & - & - & - & 1 & 1 & 1 & - & 4 \\
\hline Escápula & - & - & - & - & - & - & - & - & - & - & 6 & 2 & - & - & - \\
\hline Húmero & 2 & 1 & - & - & - & - & - & - & - & - & - & - & - & 1 & - \\
\hline Radio & 1 & - & - & - & - & - & - & - & - & - & 4 & 1 & - & 3 & - \\
\hline Ulna & - & - & - & 1 & - & - & - & - & - & - & 4 & 1 & 1 & - & 1 \\
\hline Metacarpo & 1 & - & - & - & - & 1 & - & - & - & - & 6 & - & - & - & 1 \\
\hline Pelvis & - & - & - & 1 & - & - & - & - & - & - & 4 & - & - & - & - \\
\hline Fémur & 2 & - & - & 1 & - & - & - & - & - & - & 1 & - & - & 1 & - \\
\hline Patela & - & - & - & - & - & - & - & - & - & - & - & 1 & - & - & - \\
\hline Tibia & 1 & - & - & - & - & - & 1 & - & - & - & 8 & - & 1 & - & 4 \\
\hline Astrágalo & 1 & - & - & - & - & 1 & 1 & - & - & - & 3 & - & - & 1 & - \\
\hline Calcáneo & - & - & - & - & - & - & - & - & - & - & 3 & - & - & - & - \\
\hline
\end{tabular}




\begin{tabular}{|l|r|r|r|r|r|r|r|r|r|r|r|r|r|r|r|}
\hline Metatarso & 1 & - & - & - & - & - & - & 1 & - & 1 & 3 & 1 & - & - & 1 \\
\hline Metápodo & - & - & - & - & - & - & - & - & - & - & - & - & - & - & 3 \\
\hline Sesamoideo & - & - & - & - & - & - & - & - & - & - & - & 1 & - & - & 1 \\
\hline Falange I & 4 & - & - & - & - & - & - & - & - & 1 & 6 & 1 & - & - & \\
\hline Falange II & 6 & - & - & - & 2 & - & - & - & - & 2 & 2 & - & - & - & 1 \\
\hline Falange III & 3 & - & - & - & - & - & - & - & - & - & 1 & - & - & - & 1 \\
\hline \multicolumn{1}{|c}{ Total } & $\mathbf{5 1}$ & $\mathbf{2 7}$ & $\mathbf{5}$ & $\mathbf{5 3}$ & $\mathbf{1 3 6}$ & $\mathbf{8}$ & $\mathbf{2}$ & $\mathbf{1}$ & $\mathbf{2}$ & $\mathbf{4}$ & $\mathbf{7 4}$ & $\mathbf{1 2}$ & $\mathbf{5}$ & $\mathbf{1 6}$ & $\mathbf{2 7}$ \\
\hline
\end{tabular}

\section{Índice de}

Fragmentación

0,00

0,20

0,40

0,60

0,80

UE $26-2^{\mathrm{a}} \operatorname{mitad} \mathrm{s}$. XVII

UE $27-1^{\mathrm{a}} \operatorname{mitad} \mathrm{s}$. XVII

UE $29-1^{\mathrm{a}} \operatorname{mitad} \mathrm{s}$. XVII

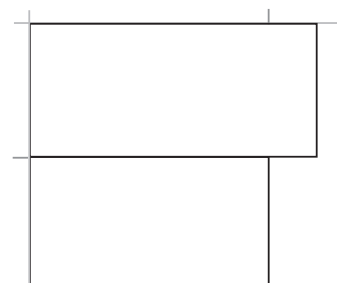

UE 48 - inicios s. XVII

UE 55 - final s. XV
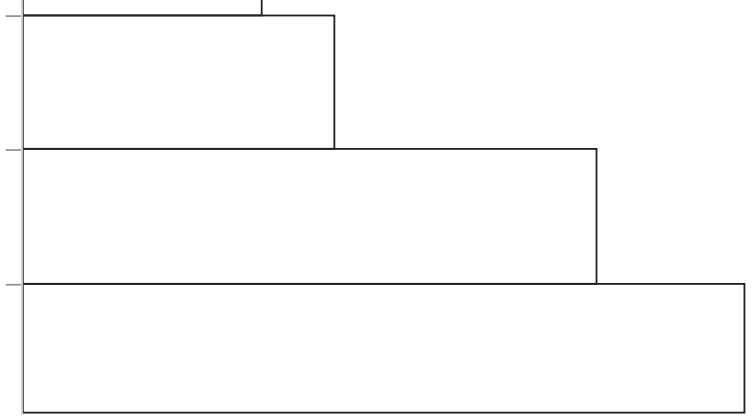

Figura 4. Valores del índice de fragmentación (IF) por unidad estratigráfica. Se han utilizado para el cálculo los datos de las especies Tipos I y II.

que estos depósitos tienen una funcionalidad de basurero donde el agente productor es el humano por la presencia de especies domesticadas. No obstante, en el análisis de la conservación de las partes anatómicas se ha detectado una particularidad, ya que en las unidades 27,48 y 55 entre un 80 y un $100 \%$ de los especímenes de bovinos pertenecen a mandíbulas y huesos craneales (tabla 7), siendo este porcentaje superior al $90 \%$ en los estratos 48 y 55 . Un aspecto que también se ha observado en los estratos 27 y 55 excavados fuera de la cata.
Por otro lado, los resultados de IF muestran diferencias entre dos conjuntos de estratos (fig. 4), estando en torno a 0,20 en los estratos 26,27 y 29 y superando el 0,48 en los denominados 48 y 55 . Los valores superiores de IF indican una mayor fragmentación de los huesos por lo que, al menos con este índice, se podría admitir dos estados de conservación distintos que podrían estar relacionados con funciones distintas de los dos grupos de estratos. Además, en lo que respecta a las marcas de uso (cortes o termoalteraciones) cabe destacar que solo en los estratos 
Tabla 8. Número mínimo de individuos de moluscos determinados por corte. En los estratos excavados fuera de los cortes A y B solo se han determinado fragmentos de ostras, un molusco con valvas grandes y fácilmente reconocibles.

\begin{tabular}{|c|c|c|c|}
\hline Especie & Sin corte & Corte A & Corte B \\
\hline Ostrea edulis & 19 & 15 & 6 \\
\hline Pecten maximus & - & 1 & \\
\hline Ruditapes decussatus & - & 4 & 2 \\
\hline Cerastoderma edulis & - & 1 & - \\
\hline Unionidae & - & 1 & 1 \\
\hline Melanopsis cariosa & - & 4 & 9 \\
\hline Physa acuta & - & - & 4 \\
\hline Theba pisana & - & 1 & 8 \\
\hline Rumina decollata & - & 2 & 6 \\
\hline Ferussacia fulliculus & - & - & 27 \\
\hline Cecilioides acicula & - & - & 343 \\
\hline Caracollina lenticula & - & - & 14 \\
\hline Mediterranea hydatina & - & - & 67 \\
\hline Cochlicella acuta & - & 2 & 3 \\
\hline Xerotricha apicina & - & 1 & 25 \\
\hline Indeterminado & - & - & 4 \\
\hline Total & 19 & 32 & 519 \\
\hline
\end{tabular}

26 y 27 se han descrito este tipo de huellas en los huesos, aunque siempre en una proporción inferior al $2 \%$ de los restos.

\subsection{Descripción de los cortes A y B. Contraste de metodología}

En la intervención arqueológica del Mercado de la Puerta de la Carne la cata excavada se dividió en dos cortes. En el corte A se extrajo el material de manera sistemática y sin cribar y en el corte $\mathrm{B}$ en el que se tamizó todo el sustrato. Además, a modo de contraste, se analizó el material recogido por los peones de la excavación en unidades estratigráficas ajenas a dicha cata. La descripción de este último conjunto de unidades ya ha sido expuesta en el apartado 4.1, sin embargo, el contenido de los cortes A y B debe exponerse de manera individualizada para poder realizar el contraste metodológico. Cabe destacar que el NMI descrito a continuación únicamente se utilizará para comparar la metodología, este valor es superior al descrito para la cata (apartado 4.1) debido a que la cantidad mínima de ejemplares se volvió a estimar después de unificar el registro de ambos cortes.

En el Corte A se han contabilizado 1.264 elementos con $6,05 \mathrm{~kg}$ de, al menos, 97 individuos. En este caso, los vertebrados están representados por un $95 \%$ de los restos, un $95 \%$ de la masa y un $68 \%$ de los ejemplares. En concreto se han determinado 65 ejemplares de vertebrados: 13 Bos taurus, seis suidos, 11 caprinos, un Canis familiaris, dos Felis catus, tres Oryctolagus cuniculus, un Rattus norvegicus, 14 Rattus rattus, un roedor indeterminado, tres Gallus domesticus, cinco aves indeterminadas y cinco peces indeterminados. Por su parte, el registro malacológico está compuesto por 32 individuos 


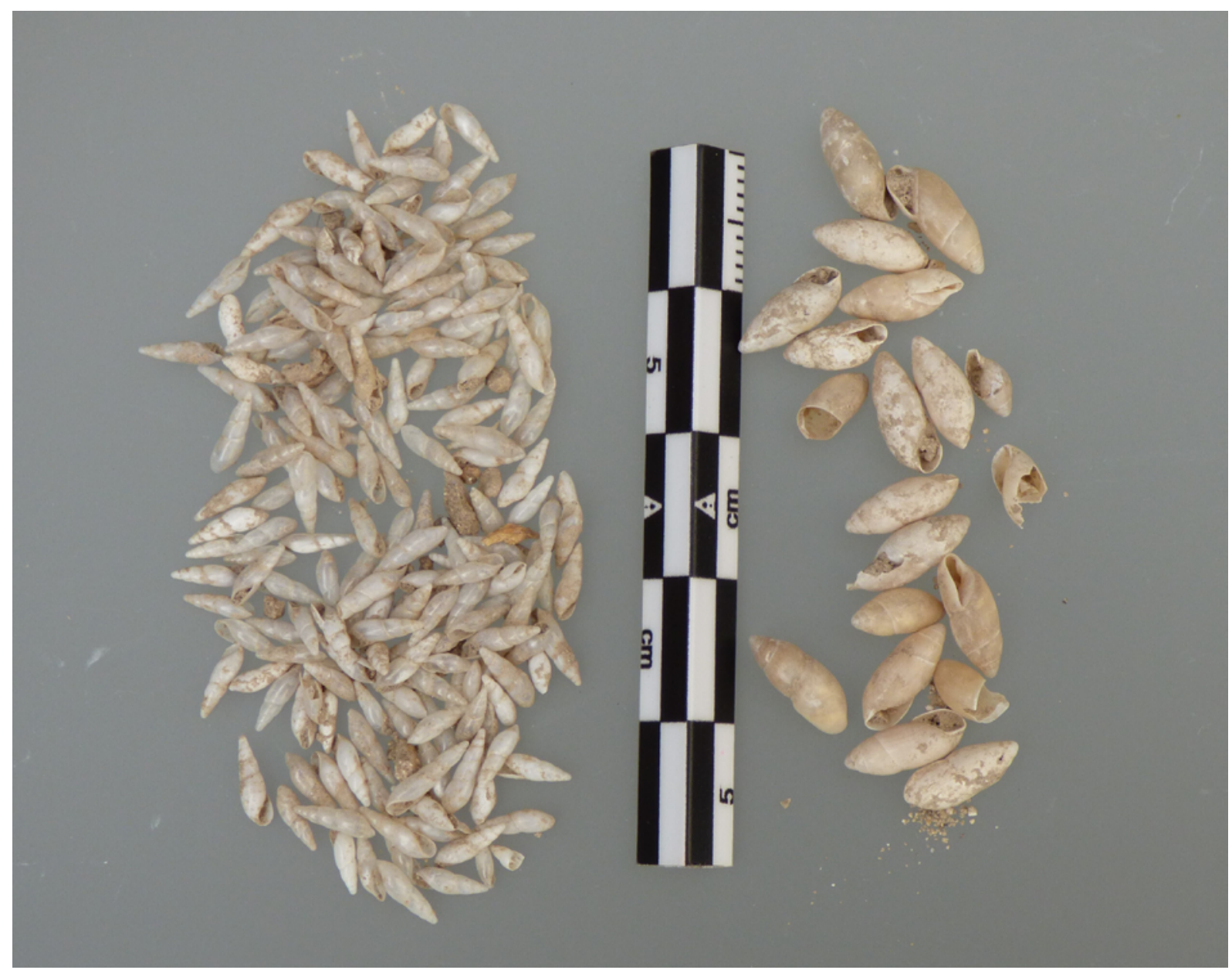

Figura 5. Detalle del conjunto malacológico rescatado en el corte B, cuyo material ha sido totalmente cribado. A la izquierda se puede observar un grupo de Mediterranea hydatina y a la derecha de Ferussacia folliculus cuya longitud (LM) es inferior a $5 \mathrm{~mm}$.

(tabla 8): 15 Ostrea edulis, un Pecten maximus, cuatro Ruditapes decussatus (almeja fina), un Cerastoderma edulis (berberecho), un ejemplar de la familia Unionidae, cuatro Melanopsis cariosa, un Xerotricha apicina, dos Cochlicella acuta y dos Rumina decollata.

En el Corte B, el registro faunístico suma 1.512 elementos, $2,16 \mathrm{~kg}$ y un NMI de 558 ejemplares. En este depósito los vertebrados suponen un $62 \%$ de los elementos, un $94 \%$ de la masa y un $8 \%$ de los ejemplares. Se han determinado 41 ejemplares de vertebrados: seis Bos taurus, un Equus caballus (caballo), tres suidos, seis caprinos, un Felis catus, un Oryctolagus cuniculus, 13 Rattus rattus, tres Gallus domesticus, siete peces indeterminados y dos anuros (ranas y sapos). El registro malacológico está compuesto por 519 individuos: seis Ostrea edulis, dos Ruditapes decussatus, un ejemplar de la familia Unionidae, nueve Melanopsis cariosa, dos
Physa acuta, nueve Theba pisana, 25 Xerotricha apicina, tres Cochlicella acuta, seis Rumina decollata, 27 Ferussacia folliculus, 343 Cecilioides acicula, 14 Caracollina lenticula, 67 Mediterranea hydatina y 4 moluscos indeterminados

\section{DISCUSIÓN}

\subsection{Contraste de metodología de rescate de material}

En la fase de excavación se llevaron a cabo tres metodologías distintas de recolección de material para demostrar que un método adecuado de rescate es esencial para un estudio arqueozoológico completo y con garantías. Aun siendo un aspecto que ya vienen apuntando otros autores (Moreno-García et al. 2003; O'Connor 


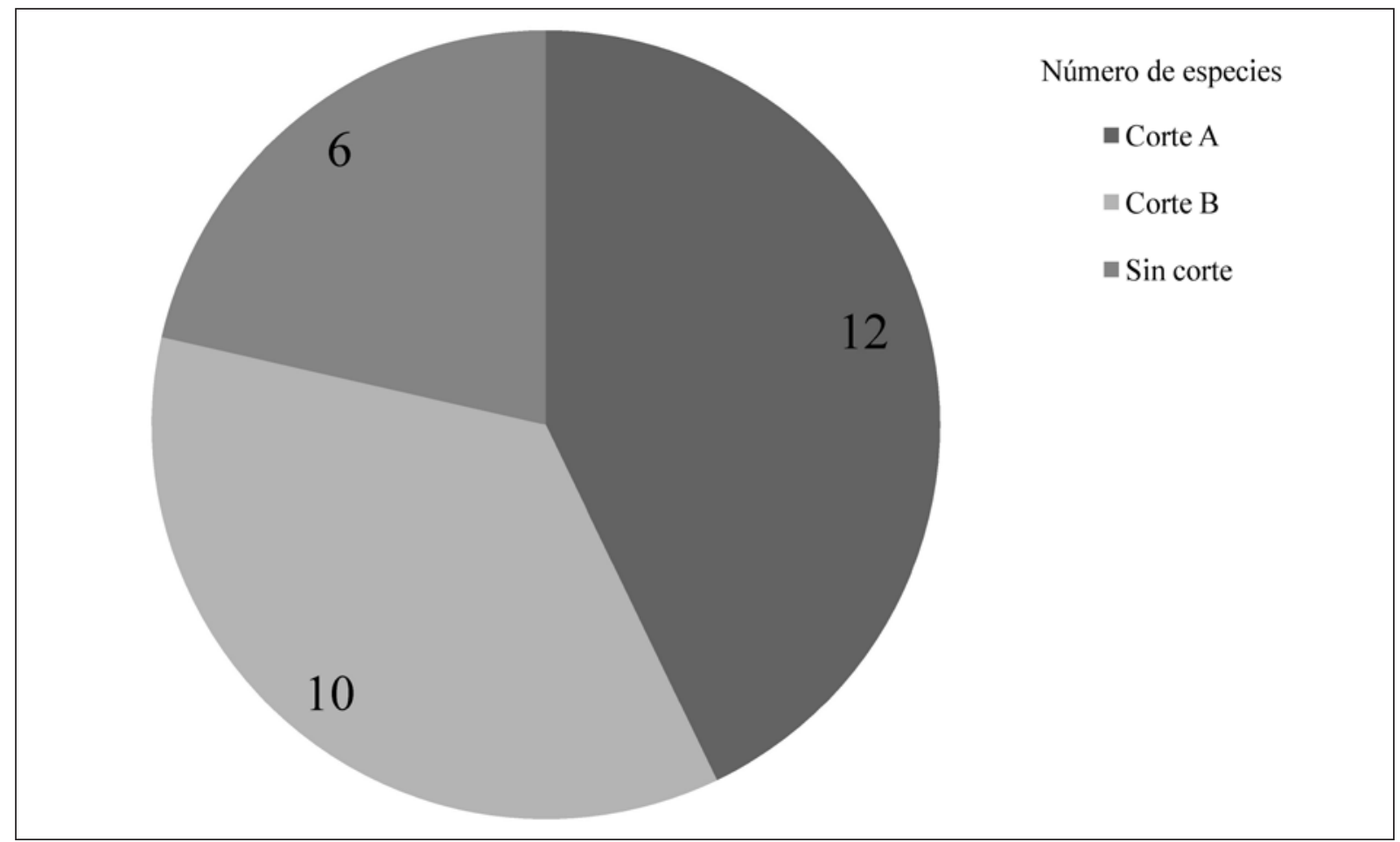

Figura 6: Porcentaje de especies de vertebrados registradas con las tres metodologías. Se observa cómo con la intervención del arqueozoólogo aumenta la probabilidad de encontrar ejemplares de especies de vertebrados con menos de $1 \mathrm{~kg}$ : seis en el corte A, cuatro en el corte B y una en las unidades ajenas a la cata (apartado 4.3).

2004), se ha considerado interesante llevar a cabo este contraste metodológico y para ello se ha seleccionado la asociación malacológica, ya que es la que presenta unas mayores diferencias en lo que respecta a la abundancia de especies e individuos determinados. Como se puede apreciar en la tabla 8, la cantidad de ejemplares rescatados en el corte $B$ (tamizado) supera en 60 veces a la registrada en corte A. Un resultado justificado por el hallazgo de gasterópodos pulmonados de pequeño tamaño (LM< $<$ mm) y en gran número (fig. 5), los cuales aportan información acerca del entorno en el que se formó el depósito. Además, es destacable que el número de especies rescatadas en los cortes A y B alcanzan la decena mientras que en las unidades en las que no participó un especialista solo se recogieron valvas de ostra plana, una especie grande y reconocible, algo que se documenta en otros yacimientos en los que se rescatan mayoritariamente restos de animales grandes según O’Connor (2004) y Grau-Sologestoa (2014).

Además, el resultado obtenido a partir del análisis del conjunto malacológico es similar al hallado con los restos de vertebrados. En la fig. 6 se puede apreciar que el número de especies de vertebrados halladas en los cortes A y B es superior al obtenido en los estratos localizados fuera de la cata, sobre todo teniendo en cuenta las especies con menos de $18 \mathrm{~kg}$ de masa corporal. Aunando estos resultados a los expuestos para la malacofauna se puede concluir que los resultados paleobiológicos de un yacimiento están totalmente relacionados con la metodología empleada durante la excavación, por lo que ésta debe ser adecuada para alcanzar unos resultados fundamentados en el total del registro faunístico de un yacimiento (Moreno-García et al. 2003; O’Connor 2004).

\subsection{Los vertebrados. Entre vacas y ratas}

En el conjunto de vertebrados determinados en este yacimiento son destacables al menos tres aspectos: una frecuencia de bovinos más alta de la esperada en relación a otras especies de ungulados, la abundancia de ratas en los estratos datados en el s. XVII y la ausencia de especies vinculadas a actividades cinegéticas.

Por un lado, destaca la abundancia de ejemplares de bovinos, estos igualan en NMI a los caprinos en 
el conjunto de los datos (tabla 1), llegando a superarlos en la unidad 48 (fig. 3). Aunque por norma general en el registro faunístico de yacimientos medievales y postmedievales vinculados a depósitos de consumo humano predominan los especímenes de caprinos, bovinos y suidos (Albarella 1999; Baldino et al. 2008), suelen ser los de caprinos los más abundantes. Este resultado podría estar relacionado con la actividad desarrollada en el matadero anejo, caracterizado por ser el único de la ciudad de Sevilla en el que sacrificaban las reses desde el s. XV, salvo excepciones como el matadero de la población judía (Bernáldez y Bernáldez 2008; Bosch 1984; Ladero 1989). De hecho, entre el 80 y el $100 \%$ de los elementos determinados pertenece a fragmentos de la zona craneal (destacando las mandíbulas y piezas dentales). Esta homogeneidad en la composición de un paleobasurero puede vincularse con una actividad especializada (Grau-Sologestoa 2014; Reitz y Wing 1999), pudiendo este tipo de vertido estar relacionado con desechos de las tenerías (Bartosiewicz 2009) y con los residuos de la sala de despojería de los mataderos (Bernáldez y Bernáldez 2008; Baldino et al. 2008). Aunque solo con este dato no se puede justificar que los estratos sean basureros in situ del matadero, sí que parece razonable que la basura esté relacionada con la actividad que se desarrollaba en el edificio contiguo. Una basura que sigue formando montículos en el entorno de la Puerta de la Carne al menos hasta el s. XVIII (Cortés 1998).

En futuras intervenciones en este mismo lugar se espera alcanzar una muestra faunística suficiente para poder describir con mayores garantías la actividad desarrollada en el matadero en lo que respecta a las características biológicas de los ejemplares sacrificados, sin embargo estos primeros resultados muestran una tendencia al sacrificio de reses con más de 24 meses de edad. Actualmente el 55\% de los bovinos en España se sacrifican con más de 12 meses (Jiménez-Montero et al. 2011), cuando ya han alcanzado el $90 \%$ de su masa corporal.

Además de los ungulados, en el yacimiento se han determinado especies de vertebrados con menos de 18 $\mathrm{kg}$ de masa corporal, aquéllos que podrían estar incluidos en los grupos de los Tipo III y IV descritos por Bernáldez et al. (2017) para mamíferos terrestres. Este tipo de especies presenta una potencialidad fósil menor que las especies enmarcadas en los grupos Tipo I y II, es decir los huesos de animales pequeños tienen menos probabilidad de llegar a formar parte del registro fósil. Según los resultados obtenidos en el Parque Nacional de Doñana el conjunto de estas especies en la tanatocenosis no representa a la comunidad ni en número de especies ni en número de ejemplares (Bernáldez 2002; 2009; 2011), por ello se deben interpretar los resultados desde el punto de vista de la ausencia o presencia de especies, más que por la frecuencia de las mismas. En muchas ocasiones el registro de estas especies está relacionado con una actividad o una situación específica, algunos ejemplos podrían ser enterramientos rápidos que evitasen el carroñeo, el hallazgo de madrigueras que produjeran acumulaciones o depósitos humanos con una funcionalidad específica. En concreto se han descrito restos de un Canis familiaris (perro), dos Felis catus (gato), tres Oryctolagus cuniculus (conejo), un Rattus norvegicus (rata europea), 26 Rattus rattus (rata negra), un roedor indeterminado, cinco Gallus domesticus (gallo), cinco aves indeterminadas, ocho peces indeterminados y dos anuros (ranas y sapos). No se han hallado marcas de corte, roeduras o termoalteraciones en ninguno de los huesos de estos ejemplares, por lo que, exceptuando a las gallinas, ninguno debió formar parte del consumo humano. En este sentido cabe destacar la ausencia de marcas de corte en los huesos de perro (un astrágalo) y de gato (un radio y dos ulnas).

En el conjunto de especies de microvertebrados destacan los roedores (tabla 1; fig. 7), los cuales se localizan en todos los estratos, incluso de manera indirecta a partir de huellas de roedura (Haglund 1992), pero sobre todo en los datados en el s. XVII. En el conjunto óseo no se han hallado esqueletos completos de rata, aunque sí que se han rescatado algunos huesos de las extremidades y vértebras caudales en conexión y casi todas las partes anatómicas están representadas. Además, no se han localizado marcas de roedura ni de erosión, por lo que se descarta que el conjunto óseo de ratas esté relacionado con la actividad trófica de un depredador. Por lo tanto, podría deducirse que probablemente los cadáveres de rata negra estaban completos en la tanatocenosis (tabla 9) y que la acumulación de ejemplares podría deberse a la muerte de un grupo in situ o a una acumulación producida por humanos después de haberlas matado. Las ratas son animales comunes en zonas urbanas (O'Connor 2000) y tradicionalmente se han relacionado con la transmisión de la enfermedad de la peste, aunque también puede propagarse por parásitos humanos e incluso a través del aire en el caso de la peste neumónica o pulmonar (Dean et al. 2018). La peste bubónica es la forma de la enfermedad que se propaga por la picadura de un piojo o pulga infectados, concretamente en el caso de la rata se debe a la pulga (Xenopsylla cheopis). Este insecto transmite la bacteria Yersinia pestis al picar a un 
Tabla 9. Partes anatómicas de rata negra conservadas en los cortes A y B.

\begin{tabular}{|c|c|c|c|c|}
\hline & UE 26 & UE 27 & UE 48 & UE 55 \\
\hline Incisivo & 5 & 6 & 7 & - \\
\hline Cráneo & - & 2 & 2 & - \\
\hline Mandíbula & 2 & 3 & 16 & - \\
\hline Costilla & 3 & - & 5 & - \\
\hline Vértebra & 3 & - & 2 & - \\
\hline Vértebra caudal & 3 & - & 17 & - \\
\hline Húmero & - & 3 & 16 & - \\
\hline Radio & 1 & - & 10 & - \\
\hline Ulna & 1 & 3 & 5 & - \\
\hline Pelvis & - & 3 & 5 & - \\
\hline Fémur & 6 & 3 & 18 & 1 \\
\hline Tibia & 7 & 7 & 6 & - \\
\hline Calcáneo & 1 & - & - & - \\
\hline Metápodo & 9 & 6 & 3 & - \\
\hline Falange I & 6 & 5 & & - \\
\hline Total & 47 & 41 & 112 & 1 \\
\hline
\end{tabular}

humano después de picar a un roedor infectado $(\mathrm{Be}-$ nedictow 2004), siendo el roedor más común la rata negra (Rattus rattus). En la ciudad de Sevilla se han descrito distintos brotes de peste a lo largo de la historia, aunque la más importante fue la de 1649, ya que produjo un descenso drástico de la población que pasó de unos 140.000 a 60.000-80.000 habitantes (Domínguez 1989; Fernández 2002). En este caso, la cantidad de ejemplares de rata negra hallada en el Mercado de la Puerta de la Carne podría tratarse de un testigo fósil de alguno de esos períodos críticos en la historia de Sevi1la. Por otro lado, el único ejemplar de rata gris (Rattus norvegicus) se ha rescatado en el estrato más moderno del s. XVII (UE 26), quizás una de las primeras evidencias de esta especie en la península ibérica, ya que se acepta la colonización europea de esta especie a partir del s. XVIII (Rojas y Palomo 2007).

Por último, en cuanto a la asociación faunística cabe destacar la ausencia de especies cinegéticas, que son comunes en otros yacimientos. Aunque se han determinado huesos de conejo, la presencia de esta especie en el registro arqueológico no está necesariamente relacionada con la alimentación, ya es una especie común en zonas de huertas y es probable que en este caso los restos óseos hallados correspondan a intrusiones naturales. El hecho de no hallar restos de ciervo, una especie cosmopolita frecuente en los yacimientos arqueológicos del suroeste de la península ibérica, podría apoyar la idea de que este basurero esté originado por la actividad del matadero.

\subsection{Los invertebrados. Consumo y caracterización ambiental}

El registro malacológico está compuesto por 619 restos de 570 ejemplares con una masa de $1,63 \mathrm{~kg}$, este dato de masa está justificado por la presencia de ostras. Por un lado, se han hallado valvas de ostra plana procedentes del consumo humano, contabilizándose 76 fragmentos de 40 ostras con una masa de 1,60 kg. El consumo de este molusco en la Sevilla moderna se ha observado en otros yacimientos de la ciudad datados desde época romana, siendo este período y el denominado "Pequeña Edad del Hielo" (siglos XIV al XVIII; Fagan 2001; Martín y Olcina 2001) los dos momentos 


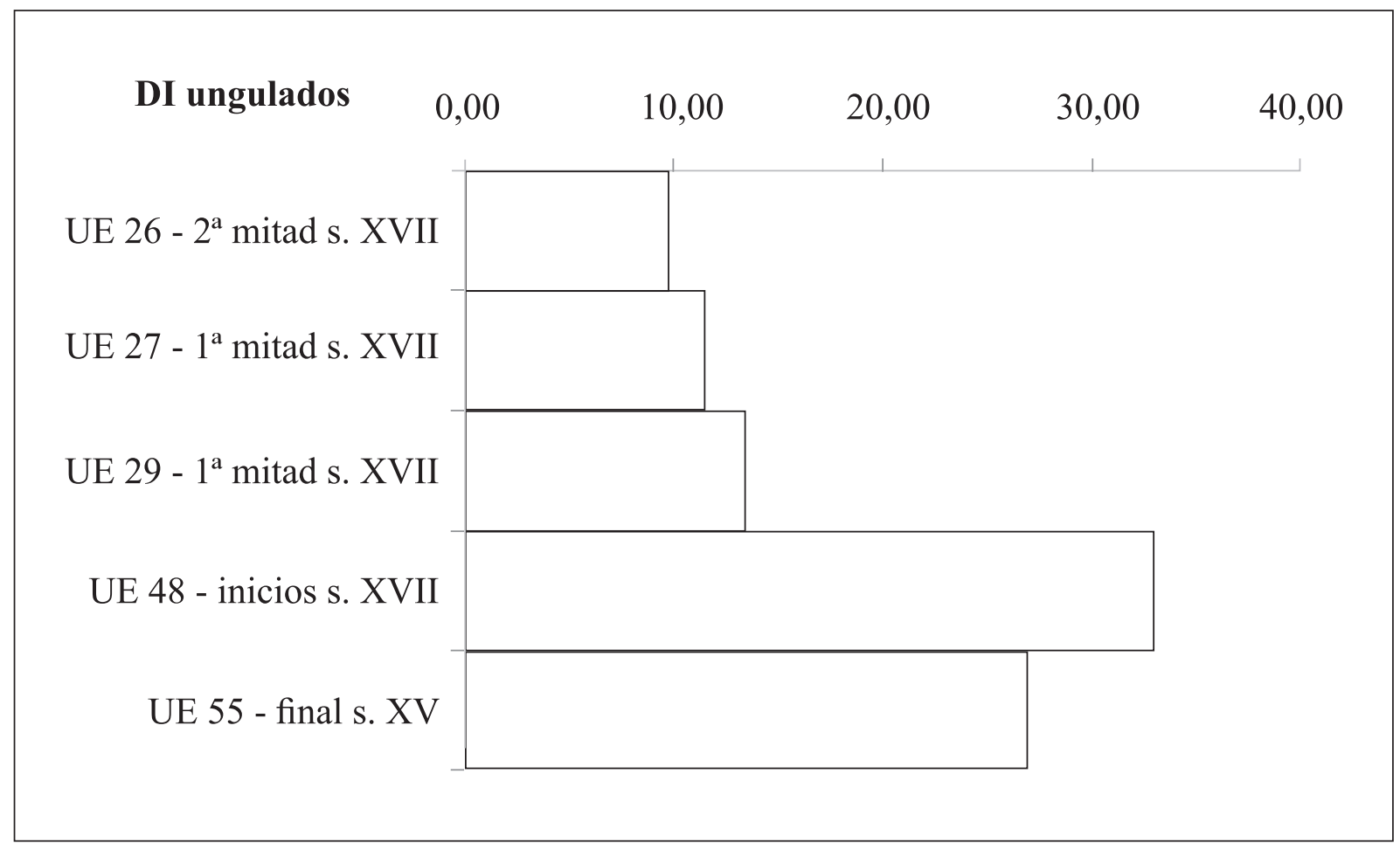

Figura 7. Muestra ósea de Rattus rattus, a la izquierda, y de Rattus Norvegicus, a la derecha, procedente de la unidad 26. La primera especie podría estar relacionada con uno de los brotes de peste documentados en la ciudad y la segunda podría ser una de las primeras evidencias descritas en el sur de la península ibérica.

con mayor frecuencia de restos de esta especie en el registro arqueológico de la ciudad. Algunos de estos yacimientos son la Plaza de la Encarnación, la Catedral, el Monasterio de Santa María de las Cuevas y las Atarazanas. Con las ostras de estos yacimientos se realizó un estudio sobre la evolución de la talla a lo largo los siglos XIV y XVIII (Bernáldez y García-Viñas 2010) en el que se observó un descenso significativo del tamaño en los individuos a finales del s. XVI y principios del s. XVII relacionado, posiblemente, con un momento de sobreexplotación de esta especie. El precio de un par de ostras era de 4 maravedíes cuando el sueldo de un estibador del muelle en el siglo XVI era de más de 300 maravedíes al día (Morales 1989), lo que demuestra que era un alimento al alcance de la población. Entre los siglos XIV al XVI según Collantes de Terán (1984) la población se triplicó, por lo que se podría considerar que este descenso en la talla de las ostras pudo ser consecuencia de una sobreexplotación, sobre todo, en la segunda mitad del siglo XVI cuando la población era de unos 140.000 habitantes (partiendo de 40.000 en el siglo XIV; Domínguez 1984; 1996). El tamaño de las ostras analizadas aumenta en el siglo XVIII, pero con una frecuencia de hallazgo muy baja. Es posible que al descender a la mitad la población, como consecuencia de la peste bubónica de 1649 (Domínguez 1989; 2003; Fernández 2002), la sobreexplotación cese, propiciando el aumento en la talla y el descenso de la frecuencia de valvas en los basureros. En este caso, el tamaño medio de los ejemplares analizados es similar a la talla estimada en los ejemplares de yacimientos de la ciudad datados en la segunda mitad del s. XVI y principios del s. XVII en los que la media de la longitud de las valvas (LM) está en torno a $77 \mathrm{~mm}$ y en el Mercado de la Puerta de la Carne en $75 \mathrm{~mm}$.

Por otro lado, el conjunto analizado de gasterópodos terrestres y de agua dulce proceden de una tanatocenosis natural y aportan información acerca de algunas condiciones microambientales de la zona de estudio durante el s. XVI y el s. XVII. Melanopsis cariosa y Physa acuta son dos moluscos de agua dulce que solo se registran en los estratos más modernos del s. XVII (UE 26 y UE 27) y que podrían indicar encharcamientos o desbordes de la fuente de agua construida en este mismo periodo y que se localiza a escasos metros de los cortes intervenidos. El resto de 


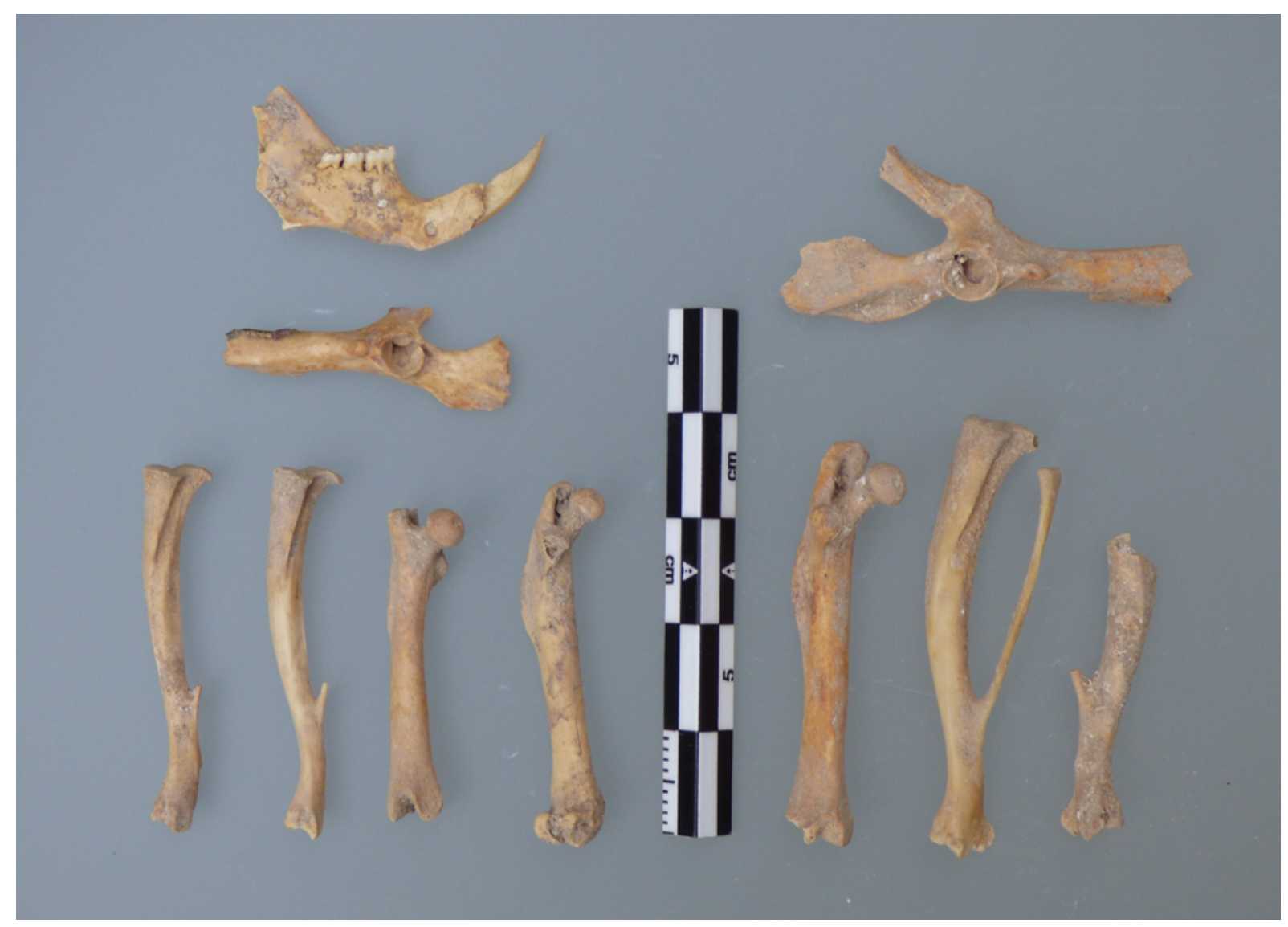

Figura 8. Densidad de individuos (DI) de ungulados por estrato $\left(\mathrm{NMI} / \mathrm{m}^{3}\right)$. Como puede observarse las unidades 48 y 55 presentan una mayor densidad de individuos, probablemente relacionada con la funcionalidad de dichos depósitos.

gasterópodos, son terrestres, destacando la presencia exclusiva de Theba pisana, Xerotricha apicina, Cochlicella acuta, Rumina decollata en el estrato datado a finales del s. XV y la dominancia en el conjunto de Ferussacia folliculus, Cecilioides acicula, Caracollina lenticula, Mediterranea hydatina en los niveles del s. XVII. Ambas asociaciones malacológicas son la evidencia de diferencias en la humedad relativa del entorno en ambos momentos.

\subsection{Estratigrafía de la basura. Función de la zona}

En el análisis funcional de los depósitos estudiados, además de las observaciones in situ y de la tipología de los estratos, se analizará la densidad de ejemplares de ungulados registrados en la cata analizada. La estratigrafía de la zona intervenida presentaba una serie de unidades estratigráficas con tendencia a la horizontalidad, con la excepción de la unidad 48 (fig. 2).
Esta última presentaba un buzamiento hacia el exterior del muro y contenía mandíbulas de bovino situadas de manera horizontal apoyadas en el suelo de cal denominado UE 30. Esta estratigrafía podría indicar que todos los estratos analizados son vertidos para enrasar distintos niveles de suelo (UU.EE. 26, 27 y 29), excepto las UU.EE. 48 y 55. La primera de éstas podría tratarse de un depósito basurero in situ atendiendo a su mayor densidad de basura (fig. 8) y de ejemplares de rata negra (fig. 9), a la morfología del estrato (en cuña, apoyado en la pared) y a la localización de mandíbulas en posición horizontal. Por su parte, la UE 55 también podría tratarse de un basurero in situ si se tiene en cuenta la densidad de ejemplares de ungulados y que la potencia real del estrato es de más de $90 \mathrm{~cm}$, una potencia que podría estar más relacionada con un muladar que con una nivelación del suelo. Además, cabe añadir que la fragmentación de los restos en las UU.EE. 48 y 55 (IF mayor a 0,48) es superior a la observada en los restantes estratos (IF 


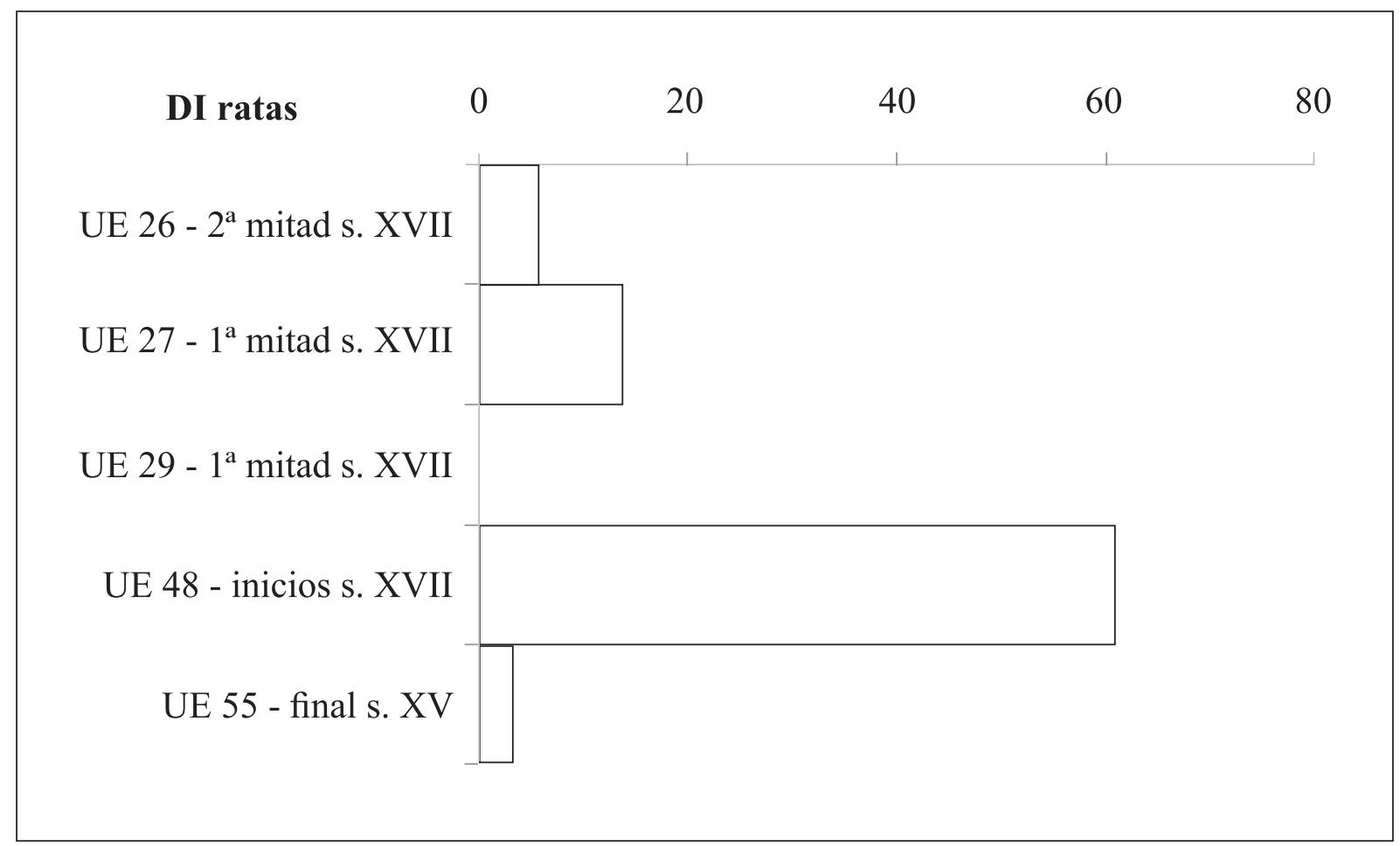

Figura 9. Densidad de individuos (DI) de rata negra $\left(\mathrm{NMI} / \mathrm{m}^{3}\right)$. Es destacable el estrato 48 , el cual podría tratarse de un basurero datado en la primera mitad del s. XVII.

en torno a 0,20$)$, lo que denota un uso distinto de ambos conjuntos de unidades estratigráficas. De hecho, analizando las marcas de uso se ha podido certificar esta diferencia al detectarse cortes y termoalteraciones únicamente en las unidades 26 y 27. Estas huellas de uso podrían relacionar a estos vertidos más con un basurero de la actividad trófica cotidiana que con los residuos de un matadero. En este sentido es interesante destacar que el estrato 26 la proporción de caprinos supera al de bovinos, una basura que no es propia de un matadero de reses. Estas últimas unidades se diferencian también en la asociación malacológica descrita, ya que está compuesta por caracoles terrestres vinculados a zonas de humedad y que viven enterradas o bajo piedras y vegetación (Cecilioides acicula, Mediterranea hydatina, Caracollina lenticula y Ferussacia folliculus) y gasterópodos de agua dulce (Melanopsis cariosa y Physa acuta). Este registro probablemente esté relacionado con la fuente anexa datada en el s. XVII, mientras que el registro de finales del s. XV (UE 55) está compuesto por especies que habitan en superficie y que están vinculadas a hábitats más secos (Theba pisana, Xerotricha apicina, Cochlicella acuta).

\section{CONCLUSIONES}

El estudio paleobiológico y tafonómico del material faunístico rescatado en la campaña de 2017 del Mercado de la Puerta de la Carne (Sevilla) ha concluido con el análisis de 2.960 restos (2.724 excluyendo las piezas dentales), $13,76 \mathrm{~kg}$ y 684 ejemplares de especies animales de vertebrados e invertebrados.

Por un lado, el conjunto de vertebrados es una evidencia de la actividad que se desarrolla en ese lugar y en esa época, destacando la alta abundancia relativa de bovinos respecto a lo conservado en otros yacimientos, así como de ratas negras. Los bovinos podrían estar relacionados con el matadero y las ratas con la insalubridad propia de la época y probablemente con algunos de los brotes de peste negra, sobre todo con la de la segunda mitad del siglo XVII que se dio entre los meses de mayo y junio de 1649 .

Por otro lado, el registro de moluscos está compuesto por especies procedentes de una tanatocenosis natural, las cuales han permitido entender cambios en el medio derivados de la instalación de una fuente; con la excepción de la ostra plana (Ostrea edulis) que podría responder al consumo humano o al uso secundario de las valvas como material de construcción. 
Los resultados paleobiológicos junto al análisis estratigráfico han permitido interpretar el proceso de colmatación del exterior del edificio del matadero, pudiendo interpretarse como muladares o basureros los dos estratos más antiguos excavados. El resto podrían tratarse de fases de relleno y reparación de la calle a partir de basureros cercanos.

Por último, se ensayaron tres metodologías de rescate del material cuyos resultados han dejado patente la importancia de la participación de los especialistas en las labores de excavación y en el diseño del muestreo.

\section{Agradecimientos}

Desde el Laboratorio de Paleobiología del Instituto Andaluz del Patrimonio Histórico (IAPH) queremos agradecer a la empresa Arqueópolis S.L. la invitación a formar parte del equipo de este proyecto en todas sus fases.

\section{BIBLIOGRAFÍA}

Albarella, U. (1999): "The mystery of husbandry': medieval animals and the problem of integrating historical and archaeological evidence". $A n-$ tiquity 79: 867-875. DOI: https://doi.org/10.1017/ S0003598X00065601

Arenas, P.; Carrasco, I.; Conlin, E.; Jiménez, A.; Lafuente, P.; Martín, A. y Vera, E. (2004): "El palacio de Hernando Colón: arqueología de la arquitectura en el patio de San Laureano (Sevilla)". Romula 3: 285-310.

Baldino, B.; Carenti, G.; Grassi, E.; Orgolesu, T.; Secchi, F. y WilkenS, B. (2008): "L'economia animale dal Medioevo all'Età moderna nella Sardegna nord occidentale". Sardinia, Corsica et Baleares antiquae: International Journal of Archaeology, VI: 103-155.

Barone, R. (1999): Anatomie comparée des mammifères domestiques, Tome 1, Ostéologie. Paris, Vigot.

Bartosiewicz, L. (2009): "Skin and Bones: Taphonomy of a Medieval Tannery in Hungary". Journal of Taphonomy 7: 91-107.

Behrensmeyer, A.K. y Boaz, D.E.D. (1980): “The Recent Bones of Ambosely National Park, Kenya in Relation to East African Paleobiology", en A.K. Behrensmeyer y A.P. Hills (eds.), Fossils in the Making: 72-92. Chicago, University Chicago Press.
Benedictow, O.J. (2004): The Black Death 1346-1353. The Complete History. Woodbridge, The Boydell Press.

Bernáldez, E. (1996): "El nicho ecológico de la paleobiología en el Patrimonio Histórico". PH: Boletín del Instituto Andaluz del Patrimonio Histórico 16: 49-59.

Bernáldez, E. (2002): "Bioestratimony of terrestrial mammals in Doñana National Park (Spain), en M. de Renzi, M.V. Pardo, M. Belinchón, E. Peñalver, P. Montoya y A. Márquez-Aliaga (eds.), Current topics on Taphonomy and fossilization: 314-324. Valencia, Ayuntamiento de Valencia.

Bernáldez, E. (2009): Bioestratinomía de macromamíferos terrestres de Doñana. Inferencias ecológicas en los yacimientos arqueológicos del S.O. de Andalucía. BAR International Series 1978. Oxford, Archaeopress.

Bernáldez, E. (2011): "Biostratinomy applied to the interpretation of scavenger activity in paleoecosystems". Quaternary International 243: 161-170. DOI. 10.1016/j.quaint.2011.06.023

Bernáldez, E. y Bernáldez, M. (2000) "La basura orgánica de Lebrija en otros tiempos: Estudios paleobiológico y tafonómico del yacimiento arqueológico de la calle Alcazaba de Lebrija". PH. Boletín del Instituto Andaluz del Patrimonio Histórico, 32: 134-150.

Bernáldez, E. y Bernáldez, M. (2002): "El subsuelo de la Catedral de los siglos XI al XVIII: De vertedero a cantera", en A. Jiménez (ed.), Magna Hispalensis (I). Recuperación de la aljama almohade: 429-472. Sevilla, Cabildo Metropolitano.

Bernáldez, E. y Bernáldez, M. (2008): “Ancient and new slaughterhouses: The "Calle Vírgenes, 9" medieval archaeological site (Seville)", en J. Aguirre, J.C. Braga, A.G. Checa, M. Company y F.J. Rodríguez-Tovar (eds.), Abstracts $3^{\text {rd }}$ Meeting in Taphonomy and Fossilization: 21-22. Granada (2008), Granada, IGME-Universidad de Granada.

Bernáldez, E. y García-Viñas, E. (2010): "Indirect detection of Seville population studying size of oysters?". Munibe, suplemento 31: 208-215.

Bernáldez, E.; García-Viñas, E.; Sánchez-Donoso, I. y Leonard, J.A. (2017): "Bone loss from carcasses in Mediterranean ecosystems". Palaios 32: 1-7.

Bosch, J. (1984): La Sevilla islámica: 712-1248. Sevilla, Universidad de Sevilla.

Collantes de Terán, A. (1984): Sevilla en la Baja Edad Media. La ciudad y sus hombres. Sevilla, Publicaciones del Ayuntamiento de Sevilla.

Cortés, J. (1998): "Sevilla extramuros. La evolución de los espacios periurbanos", en M. Valor y C. Romero 
(coords.), Sevilla extramuros: la huella de la historia en el sector oriental de la ciudad: 55-102. Sevilla, Universidad de Sevilla.

Dean, K.R.; Krauer, F.; Walløe, L.; Lingjærde, O.C.; Bramanti, B.; Stenseth, N.C. y Schmid, B.V. (2018): "Human ectoparasites and the spread of plague in Europe during the Second Pandemic". Proceedings of the National Academy of Sciences 115 (6): 13041309. https://doi.org/10.1073/pnas.1715640115.

Domínguez, A. (1984): La Sevilla del siglo XVII. Sevilla, Universidad de Sevilla.

Domínguez, A. (1989): "La población de Sevilla a mediados del siglo XVII". Archivo Hispalense: Revista Histórica, Literaria y Artística 221: 3-15.

Domínguez, A. (1996): Los extranjeros en la vida española durante el siglo XVII. Sevilla, Diputación de Sevilla.

Domínguez, A. (2003): Orto y ocaso de Sevilla. Sevilla, Diputación de Sevilla.

Fagan, B. (2001): The Little Ice Age: How climate made History. Santa Barbara, Basic Books.

Fernández, V. (2002): "Luces de modernidad. De la Sevilla amurallada a la metropolitana", en Edades de Sevilla: Hispalis, Isbiliya, Sevilla: 101-120. Sevilla, Excmo. Ayuntamiento de Sevilla.

Fisher, D.C. (1995): "Bone surface modifications in zooarchaeology". Journal of Archaeological Method and Theory 2: 7-68.

García-Viñas, E. y Bernáldez, E. (2013) “Paleobiología en Andalucía: una primera aproximación a los estudios arqueozoológicos realizados para la Prehistoria Reciente", en J. Jiménez; M. Bustamante y M. García (eds.), Actas del VI Encuentro de Arqueología del Suroeste Peninsular: 897-926. Villafranca de los Barros (2012), Excmo. Ayuntamiento de Villafranca de los Barros.

Grau-Sologestoa, I. (2014): "Waste management at early medieval rural sites of northern Spain: Taphonomic issues for interpreting faunal remains". Quaternary International 330: 97-108. DOI: https://doi. org/10.1016/j.quaint.2013.12.016

Haglund, W.D. (1992): "Contribution of rodents to postmortem artifacts of bones and soft tissue". Journal of Forensic Sciences, 37: 1459-1465.

Jiménez, A. (2017): Informe preliminar. Actividad arqueológica preventiva en el Mercado de la Puerta de la Carne. (Sevilla). Informe inédito depositado en el Delegación Provincial de la Consejería de Cultura.
Jiménez-Montero, J.A.; González-Recio, O. y Alenda, R. (2011). "Consideraciones sobre el nivel productivo del ganado vacuno de carne en España". Tierras de Castilla y León. Ganadería 183: 46-49.

Ladero, M.A. (1989): La Ciudad Medieval (1298-1492). Sevilla, Publicaciones de la Universidad de Sevilla.

Lyman, R.L. (1994): Vertebrate taphonomy. Cambridge, Cambridge University Press.

Martín, J. y Olcina, J. (2001): Climas y tiempos de España. Madrid, Alianza Editorial.

Morales, F. (1989): Historia de Sevilla: la ciudad del quinientos. Sevilla, Editorial Universidad de Sevilla.

Morales, A.; Cereijo, M.A.; Brännstön, P. y Liesau, C. (1994): The mammals. Castillo de Doña Blanca. Archaeoenvironmental investigations in the Bay of Cádiz, Spain (750-500 B.C.), BAR International Series 593: 37-70. Oxford, John Hedges.

Moreno-García, M.; Davis, S.D. y Pimenta, C. (2003): "Arqueozoologia:estudo da fauna no passado", en J.E. Mateus y M. Moreno-García, M. (eds.), Trabalhos de Arqueologia, 29 - Paleoecologia Humana e Arqueociências. Um programa multidisciplinar para a Arqueologia sob a tutela da cultura: 191-234

O'Connor, T. (2004): The archaeology of animal bones. Gloucestershire, Phoenix Mill.

Reitz, E.J. y Wing, E.S. (1999): Zooarchaeology. Cambridge, Cambridge University Press.

Riquelme, J.A. (1998): Contribución al estudio arqueofaunistico durante el Neolítico y la Edad del Cobre en las Cordilleras Béticas: el yacimiento arqueológico de Los Castillejos en Las Peñas de los Gitanos, Montefrio (Granada). Tesis Doctoral, Universidad de Granada. Inédita.

Rojas, A.B. y Palomo, L.J. (2007): “Rattus norvegicus (Berkenhout, 1769)”, en L.J. Palomo; J. Gisbert y J.C. Blanco (Coord), Atlas y libro rojo de los mamíferos terrestres de España: 458-459. Madrid, MAGRAMA.

Rowley-Conwy, P.; Albarella, U. y Dobney, K. (2012), "Distinguishing wild boar from domestic pigs in Prehistory: A review of approaches and recent results", Journal of World Prehistory 25: 1-44. DOI: https://doi.org/10.1007/s10963-012-9055-0.

Schmid, E. (1972): Atlas of animal bones for prehistorians, archaeologists and quaternary geologists. Amsterdam, Elsevier.

Yravedra, J. (2006): Tafonomía aplicada a Zooarqueología. Madrid, UNED ediciones. 